
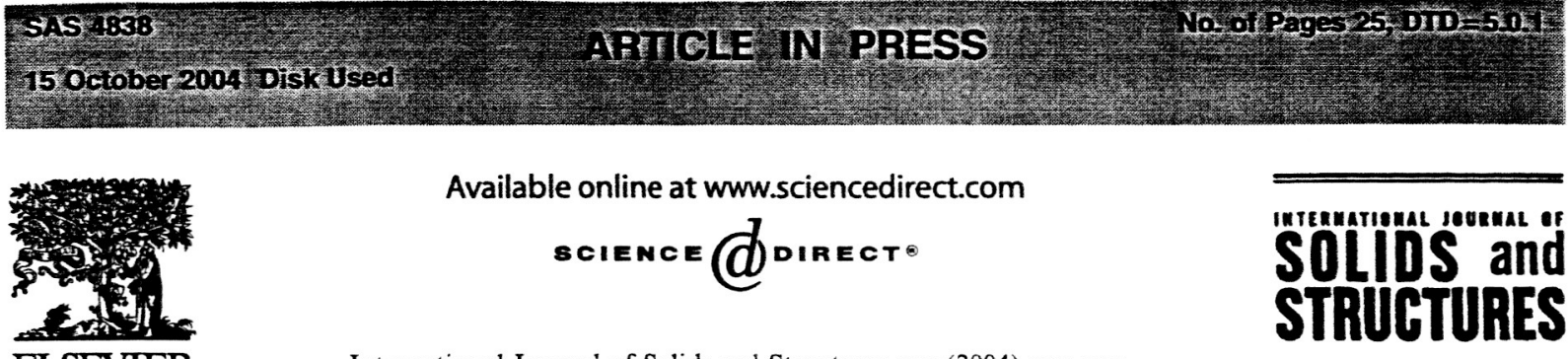

Available online at www.sciencedirect.com

science 0 Directo

ELSEVIER

International Journal of Solids and Structures xxx (2004) xxx-xxx

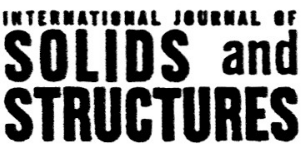

www.elsevier.com/locate/ijsolstr

2

3

4

5

6

9

\title{
Abstract
}

\section{Numerical and experimental dynamic characteristics of thin-film membranes}

\author{
Leyland G. Young, Suresh Ramanathan, Jiazhu Hu, P. Frank Pai * \\ Department of Mechanical and Aerospace Engineering, University of Missouri-Columbia, E2403C Engineering Buld. \\ East, Columbia, MO 65211, USA
}

Received 16 September 2004

\begin{abstract}
Presented is a total-Lagrangian displacement-based non-linear finite-element model of thin-film membranes for static and dynamic large-displacement analyses. The membrane theory fully accounts for geometric non-linearities. Fully non-linear static analysis followed by linear modal analysis is performed for an inflated circular cylindrical Kapton membrane tube under different pressures, and for a rectangular membrane under different tension loads at four corners. Finite-element results show that shell modes dominate the dynamics of the inflated tube when the inflation pressure is low, and that vibration modes localized along four edges dominate the dynamics of the rectangular membrane. Numerical dynamic characteristics of the two membrane structures were experimentally verified using a Polytec PI PSV-200 scanning laser vibrometer and an EAGLE-500 8-camera motion analysis system.

(C) 2004 Published by Elsevier Ltd.

Keywords: Membrane dynamic characteristics; Non-linear finite elements; Scanning laser vibrometer; Camera-based motion analysis system
\end{abstract}

\section{Introduction}

Recently there has been a renewed interest in deployable/inflatable structures for terrestrial investigations. Moreover, because the cargo space of a launch vehicle is always limited, large space structures must be designed to be stowed during launch and deployed once on orbit. Hence, instead of using previous electro-mechanical deployment systems, recent efforts of NASA concentrate on the use of inflatable structures for space applications (Salama et al., 2000; Jenkins, 2001). For example, NASA conducted the Inflatable

\footnotetext{
* Corresponding author. Tel.: +573884 1474; fax: +57 38845090.

E-mail address: paip@missouri.edu (P.F. Pai).
} 


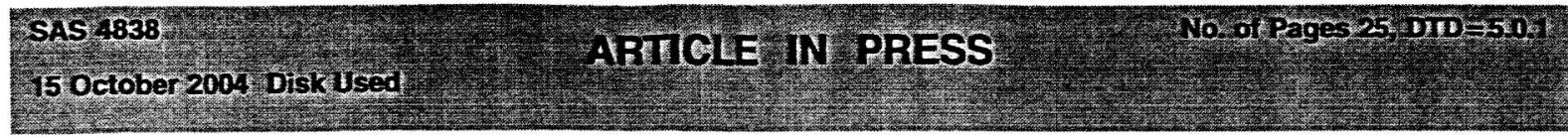

Antenna Experiment (IAE) in 1996 (Dornhiem and Anselmo, 1996). The membrane antenna having an inflated diameter of $50 \mathrm{ft}$ with three $93 \mathrm{ft}$ long struts was transported by the space shuttle Endeavour in a $7 \mathrm{ft} \times 3 \mathrm{ft} \times 1.5 \mathrm{ft}$ container. Also inflatable membrane structures have been used in parabolic antennas, radiators, solar concentrators, sun shields, habitats, radio-frequency structures, optical communication systems, radars, lightweight radio-meters, telescopes, etc. Moreover, large balloons are also membrane structures that have been used for many scientific missions. Advantages of membrane structures include smallstowed volume, lightweight, low cost, and good thermal and damping properties (Palisoc, 1994). However, there are difficulties in the design of large scientific membranes (Damle et al., 1997).

Over the last few decades, studying the dynamic behaviors of inflatable membrane structures has proven to be a challenging job. Many researchers have studied the dynamic characterization of membranes using numerical methods and, when possible, experimental approaches. Numerical methods such as finite difference and boundary elements were used by some researchers to compute vibration modes and frequencies of inflatable dams (Hsieh and Plaut, 1990). The membrane material used in the numerical analysis was assumed inextensible and its weight was neglected in the determination of the equilibrium shape. They found that the membrane's mass density is of little influence on the computed natural frequencies. Other researchers used finite elements and boundary elements to model and compute natural frequencies and mode shapes of a single-anchor inflatable dam (Mysore and Liapis, 1998). They found that the rigid foundation that anchors the dam increases the frequencies whereas the presence of impounded water tends to reduce the frequencies. They noted that the natural frequencies are dependent on the internal pressure as well as the hydrodynamic pressure of the impounding water. The pressure in an inflatable structure can also play a critical role in the suppression of vibration (Choura, 1997). This study found that the vibration suppression of inflatable structures can be accomplished by varying the internal pressure and thus there is no need of other external actuators for vibration suppression.

Some researchers tested extremely lightweight inflatable structures in a vacuum chamber and in the ambient atmospheric condition (Slade et al., 2001). They found a lack of correlation between the two cases, and they explained it to be caused by air damping. Because the coupling of a lightweight membrane and air is a highly non-linear and localized fluid-structure interaction problem, it is difficult to perform accurate numerical modeling and simulation of such problems. Hence, testing inflatable structures in vacuum conditions becomes necessary in order to verify numerical predictions. Moreover, because of the size limitation on actual vacuum chambers, tests in vacuum conditions for large membrane structures are only possible by using scaled models (Pappa et al., 2001). Johnson and Lienard (2001) obtained the natural frequencies and mode shapes of a one-tenth scale Next Generation Space Telescope (NGST) using a finite-element model developed using the cable network method. The difference between predicted and measured natural frequencies ranges from $2 \%$ to $27 \%$, and it was noted that predicted mode shapes correlated well for strutdominated modes, while membrane-dominated modes showed less correlation. The study of pre-stressed membranes by Hall et al. (2002) showed that the natural frequencies in air are lower than the ones in vacuum because air acts as a non-structural mass. But, the numerical natural frequencies obtained by Kukathasan and Pellegrino (2002) were lower than experimental vacuum ones and the error was attributed to an inaccurate tension force or Young's modulus. However, they stated that the error reduced as the tension force was increased. Experiments also showed that it is difficult to excite global vibration modes of a membrane structure by applying excitations at inflatable components because these components have high local flexibility, and resonant frequencies may vary with the excitation location (Pappa et al., 2001; Gaspar et al., 2002). Moreover, because the light weight of membranes, contact sensors cannot be used in testing and non-contact sensors (e.g., scanning laser vibrometers) need to be used (Gaspar et al., 2002).

In recent years many researchers used commercial finite-element packages to model and analyze non-linear elastic problems of thin-thickness membrane structures (Wong and Pellegrino, 2003; Kukathasan and Pellegrino, 2003; Johnston and Parrish, 2003; Jha and Inman, 2003; Holland et al., 2003; Greschik et al., 2003). Because of thin thickness, how to prevent wrinkling becomes the major task in the design of mem- 
76

brane structures (especially those for communication use), and hence membrane wrinkling has been heavily studied (Wong and Pellegrino, 2003; Kukathasan and Pellegrino, 2003; Jacobson et al., 2004; Su et al., 2003; Sutjahjo et al., 2004). Other important problems studied include air mass effect (Giraudeau et al., 2002; Kukathasan and Pellegrino, 2003), deployment methods and dynamics (Tsunoda et al., 2003), stiffening by internal pressure, follower-force effect by internal pressure (Jha and Inman, 2003), wrinkle-free design (Sakamoto et al., 2003), crease of membranes, curling of membrane edges caused by residue stresses, gravity-induced sag and pre-stressing (Jacobson et al., 2004), local buckling due to defects, clefting (Lennon and Pellegrino, 2000), and experimental techniques for accurate non-contact measurements (Slade et al., 2003; Bales et al., 2003). In wrinkling analysis, the major task is to predict wrinkling regimes, out-of-plane displacements, wrinkle wavelengths, and dynamic characteristics after wrinkling (Wong and Pellegrino, 2003; Kukathasan and Pellegrino, 2003). Because of thin thickness, such analysis always requires the use of very small plate or shell elements and hence very long computation time is needed. One can see from the literature that ABAQUS (2001) is one of the popular finite-element codes used in solving such non-linear elastic problems (Wong and Pellegrino, 2003; Kukathasan and Pellegrino, 2003; Johnston and Parrish, 2003; Jha and Inman, 2003). However, plate and shell elements in commercial codes usually do not include effects that are special to membranes, such as air mass effect and pressure-induced follower-force effect. Moreover, dynamic analysis of post-buckled structures requires a fully non-linear static analysis and then a linear modal analysis, and it usually requires special attentions in using commercial codes because different remedial techniques are used for improving convergence and accuracy, preventing numerical singularity, and/or accounting for large geometric non-linearities. Hence, even the post-buckling analysis results from popular commercial codes do not always match with experimental data (Kukathasan and Pellegrino, 2003; Jha and Inman, 2003). Furthermore, although some commercial packages can give somewhat reasonable results, the black-box feeling in using those remedial techniques of commercial packages is always a hidden pain of researchers. For university professors it is especially painful because it is costly and inconvenient to use commercial packages for teaching finite-element courses, especially the second finite element course. Hence, researchers have been improving their in-house finite-element codes by adding new non-linear elements and solution sequences for analyzing membrane structures (Jacobson et al, 2004; Su et al., 2003; Sutjahjo et al., 2004).

The above review shows that study of membranes is challenging because of the modeling, analysis, and experimental issues caused by the light weight, high flexibility, thin thickness, and air mass effect. This work is to avoid some of these issues by developing a non-linear membrane element by implementing a fully nonlinear membrane theory, to use a scanning laser vibrometer and a motion analysis system for dynamic testing, and to investigate the dynamic characteristics of thin-film membranes subjected to internal pressures and/or external tension loads.

\section{Theoretical background}

Fig. 1 shows the three coordinate systems needed for the modeling of initially curved membranes. The $x y z$ is an orthogonal curvilinear coordinate system with the curvilinear axes $x$ and $y$ being on the undeformed reference surface of the membrane and the $z$ axis being a rectilinear axis, and the $\xi \eta \zeta$ is an orthogonal curvilinear coordinate system with the curvilinear axes $\xi$ and $\eta$ being on the deformed reference surface and the $\zeta$ axis being a rectilinear axis. The $\hat{\xi}$ and $\hat{\eta}$ represent the convected configurations of $x$ and $y$ axes. Also, an inertial rectangular coordinate system $a b c$ is used for reference purpose in the calculation of initial curvatures. The $\mathbf{i}_{k}$ are unit vectors along the $\xi, \eta, \zeta$ axes, $\mathbf{j}_{k}$ are unit vectors along the $x, y, z$ axes, and $\mathbf{i}_{a}, \mathbf{i}_{b}$, and $\mathbf{i}_{c}$ are unit vectors along the $a, b, c$ axes. It can be shown that the variations of extension strains $e_{1}$ and $e_{2}$ and shear strain $\gamma_{6}\left(=\gamma_{61}+\gamma_{62}\right)$ on the reference plane of the membrane are given by (Pai and Young, 2003; Nayfeh and Pai, 2004): 

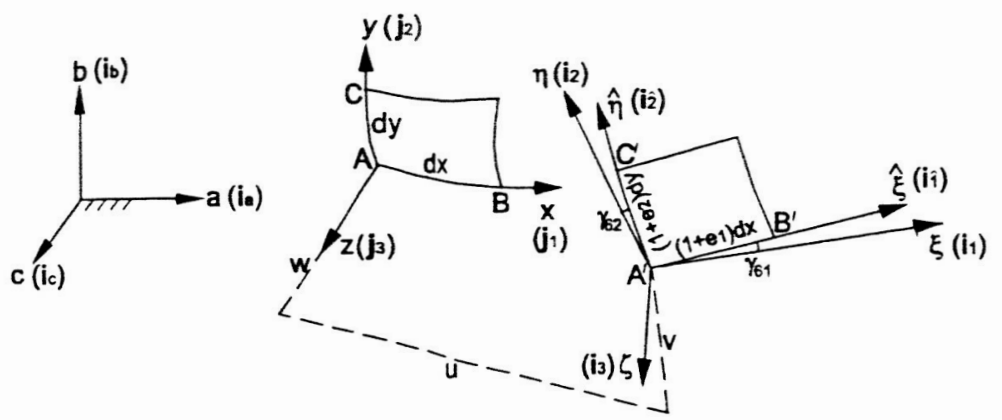

Fig. 1. The three coordinate systems for modeling and the undeformed and deformed geometries of a differential reference surface of a membrane.

$$
\begin{aligned}
\delta e_{1}= & \widehat{T}_{11} \delta t_{11}+\widehat{T}_{12} \delta t_{12}+\widehat{T}_{13} \delta t_{13} \\
\delta e_{2}= & \widehat{T}_{21} \delta t_{21}+\widehat{T}_{22} \delta t_{22}+\widehat{T}_{23} \delta t_{23} \\
\delta \gamma_{6}= & \frac{\left(\widehat{T}_{21}-\sin \gamma_{6} \widehat{T}_{11}\right) \delta t_{11}+\left(\widehat{T}_{22}-\sin \gamma_{6} \widehat{T}_{12}\right) \delta t_{12}+\left(\widehat{T}_{23}-\sin \gamma_{6} \widehat{T}_{13}\right) \delta t_{13}}{\cos \gamma_{6}\left(1+e_{1}\right)} \\
& +\frac{\left(\widehat{T}_{11}-\sin \gamma_{6} \widehat{T}_{21}\right) \delta t_{21}+\left(\widehat{T}_{12}-\sin \gamma_{6} \widehat{T}_{22}\right) \delta t_{22}+\left(\widehat{T}_{13}-\sin \gamma_{6} \widehat{T}_{23}\right) \delta t_{23}}{\cos \gamma_{6}\left(1+e_{2}\right)} \\
\delta \gamma_{61}= & \frac{\left(1+e_{2}\right) \cos \gamma_{62} \delta \gamma_{6}-\sin \gamma_{61} \delta e_{1}+\sin \gamma_{62} \delta e_{2}}{\left(1+e_{1}\right) \cos \gamma_{61}+\left(1+e_{2}\right) \cos \gamma_{62}} \\
\delta \gamma_{62}= & \frac{\left(1+e_{1}\right) \cos \gamma_{61} \delta \gamma_{6}+\sin \gamma_{61} \delta e_{1}-\sin \gamma_{62} \delta e_{2}}{\left(1+e_{1}\right) \cos \gamma_{61}+\left(1+e_{2}\right) \cos \gamma_{62}}
\end{aligned}
$$

136 where

$$
\begin{aligned}
& e_{1}=\sqrt{\left(1+u_{x}-v k_{5}^{0}+w k_{1}^{0}\right)^{2}+\left(v_{x}+u k_{5}^{0}+w k_{61}^{0}\right)^{2}+\left(w_{x}-u k_{1}^{0}-v k_{61}^{0}\right)^{2}}-1 \\
& e_{2}=\sqrt{\left(u_{y}-v k_{4}^{0}+w k_{62}^{0}\right)^{2}+\left(1+v_{y}+u k_{4}^{0}+w k_{2}^{0}\right)^{2}+\left(w_{y}-u k_{62}^{0}-v k_{2}^{0}\right)^{2}}-1 \\
& \gamma_{6}=\gamma_{61}+\gamma_{62}=\sin ^{-1}\left(\widehat{T}_{11} \widehat{T}_{21}+\widehat{T}_{12} \widehat{T}_{22}+\widehat{T}_{13} \widehat{T}_{23}\right) \\
& \gamma_{61}=\tan ^{-1} \frac{\left(1+e_{2}\right) \sin \gamma_{6}}{1+e_{1}+\left(1+e_{2}\right) \cos \gamma_{6}}, \quad \gamma_{62}=\tan ^{-1} \frac{\left(1+e_{1}\right) \sin \gamma_{6}}{1+e_{2}+\left(1+e_{1}\right) \cos \gamma_{6}} \\
& \widehat{T}_{11}=\frac{1+u_{x}-v k_{5}^{0}+w k_{1}^{0}}{1+e_{1}}, \quad \widehat{T}_{12}=\frac{v_{x}+u k_{5}^{0}+w k_{61}^{0}}{1+e_{1}}, \quad \widehat{T}_{13}=\frac{w_{x}-u k_{1}^{0}-v k_{61}^{0}}{1+e_{1}} \\
& \widehat{T}_{21}=\frac{u_{y}-v k_{4}^{0}+w k_{62}^{0}}{1+e_{2}}, \quad \widehat{T}_{22}=\frac{1+v_{y}+u k_{4}^{0}+w k_{2}^{0}}{1+e_{2}}, \quad \widehat{T}_{23}=\frac{w_{y}-u k_{62}^{0}-v k_{2}^{0}}{1+e_{2}} \\
& \delta t_{11}=\delta\left(1+u_{x}-v k_{5}^{0}+w k_{1}^{0}\right)=\delta u_{x}-k_{5}^{0} \delta v+k_{1}^{0} \delta w \\
& \delta t_{12}=\delta\left(v_{x}+u k_{5}^{0}+w k_{66}^{0}\right)=\delta v_{x}+k_{5}^{0} \delta u+k_{61}^{0} \delta w \\
& \delta t_{13}=\delta\left(w_{x}-u k_{1}^{0}-v k_{61}^{0}\right)=\delta w_{x}-k_{1}^{0} \delta u-k_{61}^{0} \delta v \\
& \delta t_{21}=\delta\left(u_{y}-v k_{4}^{0}+w k_{62}^{0}\right)=\delta u_{y}-k_{4}^{0} \delta v+k_{62}^{0} \delta w \\
& \delta t_{22}=\delta\left(1+v_{y}+u k_{4}^{0}+w k_{2}^{0}\right)=\delta v_{y}+k_{4}^{0} \delta u+k_{2}^{0} \delta w \\
& \delta t_{23}=\delta\left(w_{y}-u k_{62}^{0}-v k_{2}^{0}\right)=\delta w_{y}-k_{62}^{0} \delta u-k_{2}^{0} \delta v
\end{aligned}
$$


140 Here $k_{i}^{0}$ are initial curvatures, $u, v, w$ are displacement components of an arbitrary point on the reference

141 plane, and $u_{x} \equiv \partial u / \partial x, u_{y} \equiv \partial u / \partial y$, etc. The variations of deformed curvatures $k_{i}$ are given by

$$
\begin{aligned}
& \left\{\begin{array}{c}
-\delta k_{61} \\
\delta k_{1} \\
\delta k_{5}
\end{array}\right\}=\frac{\partial}{\partial x}\left\{\begin{array}{c}
\delta \theta_{1} \\
\delta \theta_{2} \\
\delta \theta_{3}
\end{array}\right\}-\left[K_{1}\right]\left\{\begin{array}{c}
\delta \theta_{1} \\
\delta \theta_{2} \\
\delta \theta_{3}
\end{array}\right\} \\
& \left\{\begin{array}{c}
-\delta k_{2} \\
\delta k_{62} \\
\delta k_{4}
\end{array}\right\}=\frac{\partial}{\partial y}\left\{\begin{array}{c}
\delta \theta_{1} \\
\delta \theta_{2} \\
\delta \theta_{3}
\end{array}\right\}-\left[K_{2}\right]\left\{\begin{array}{c}
\delta \theta_{1} \\
\delta \theta_{2} \\
\delta \theta_{3}
\end{array}\right\}
\end{aligned}
$$

146 where

$$
\begin{aligned}
& \delta \theta_{1}=\frac{\cos \gamma_{61}}{\cos \gamma_{6}\left(1+e_{2}\right)}\left(T_{31} \delta t_{21}+T_{32} \delta t_{22}+T_{33} \delta t_{23}\right)-\frac{\sin \gamma_{62}}{\cos \gamma_{6}\left(1+e_{1}\right)}\left(T_{31} \delta t_{11}+T_{32} \delta t_{12}+T_{33} \delta t_{13}\right) \\
& \delta \theta_{2}=\frac{\sin \gamma_{61}}{\cos \gamma_{6}\left(1+e_{2}\right)}\left(T_{31} \delta t_{21}+T_{32} \delta t_{22}+T_{33} \delta t_{23}\right)-\frac{\cos \gamma_{62}}{\cos \gamma_{6}\left(1+e_{1}\right)}\left(T_{31} \delta t_{11}+T_{32} \delta t_{12}+T_{33} \delta t_{13}\right) \\
& \delta \theta_{3}=\frac{1}{2}\left(\delta \gamma_{62}-\delta \gamma_{61}\right)+\frac{\left(\widehat{T}_{21}-\sin \gamma_{6} \widehat{T}_{11}\right) \delta t_{11}+\left(\widehat{T}_{22}-\sin \gamma_{6} \widehat{T}_{12}\right) \delta t_{12}+\left(\widehat{T}_{23}-\sin \gamma_{6} \widehat{T}_{13}\right) \delta t_{13}}{2 \cos \gamma_{6}\left(1+e_{1}\right)} \\
& -\frac{\left(\widehat{T}_{11}-\sin \gamma_{6} \widehat{T}_{21}\right) \delta t_{21}+\left(\widehat{T}_{12}-\sin \gamma_{6} \widehat{T}_{22}\right) \delta t_{22}+\left(\widehat{T}_{13}-\sin \gamma_{6} \widehat{T}_{23}\right) \delta t_{23}}{2 \cos \gamma_{6}\left(1+e_{2}\right)} \\
& {\left[K_{1}\right] \equiv\left[\begin{array}{ccc}
0 & k_{5} & -k_{1} \\
-k_{5} & 0 & -k_{61} \\
k_{1} & k_{61} & 0
\end{array}\right], \quad\left[K_{2}\right] \equiv\left[\begin{array}{ccc}
0 & k_{4} & -k_{62} \\
-k_{4} & 0 & -k_{2} \\
k_{62} & k_{2} & 0
\end{array}\right]} \\
& T_{31}=\left(\widehat{T}_{12} \widehat{T}_{23}-\widehat{T}_{13} \widehat{T}_{22}\right) / R_{0}, \quad T_{32}=\left(\widehat{T}_{13} \widehat{T}_{21}-\widehat{T}_{11} \widehat{T}_{23}\right) / R_{0}, \quad T_{33}=\left(\widehat{T}_{11} \widehat{T}_{22}-\widehat{T}_{12} \widehat{T}_{21}\right) / R_{0} \\
& R_{0} \equiv\left[\left(\widehat{T}_{12} \widehat{T}_{23}-\widehat{T}_{13} \widehat{T}_{22}\right)^{2}+\left(\widehat{T}_{13} \widehat{T}_{21}-\widehat{T}_{11} \widehat{T}_{23}\right)^{2}+\left(\widehat{T}_{11} \widehat{T}_{22}-\widehat{T}_{12} \widehat{T}_{21}\right)^{2}\right]^{1 / 2}=\left|\cos \gamma_{6}\right|
\end{aligned}
$$

155 Using the polar decomposition Jaumann strains $B_{m n}$ can be shown to be (Pai and Palazotto, 1995)

$$
B_{m n}=\frac{1}{2}\left(\frac{\partial \mathbf{u}}{\partial x_{m}} \cdot \mathbf{i}_{n}+\frac{\partial \mathbf{u}}{\partial x_{n}} \cdot \mathbf{i}_{m}\right)
$$

159 where $\mathbf{u}$ is the local displacement vector relative to the deformed coordinate system $\xi \eta \zeta$, and $x_{1} \equiv x, x_{2} \equiv y$, 160 and $x_{3} \equiv z$. The fully non-linear strain-displacement relations can be derived by using the concept of local 161 relative displacements and Fig. 1 to be (Pai and Palazotto, 1995)

$$
\{B\}=\{\psi\}
$$

165 where the change of strains through the thin thickness of a membrane is neglected and

$$
\begin{aligned}
& \{B\} \equiv\left\{B_{11}, B_{22}, 2 B_{12}\right\}^{\mathrm{T}} \\
& \{\psi\} \equiv\left\{\left(1+e_{1}\right) \cos \gamma_{61}-1,\left(1+e_{2}\right) \cos \gamma_{62}-1,\left(1+e_{1}\right) \sin \gamma_{61}+\left(1+e_{2}\right) \sin \gamma_{62}\right\}^{\mathrm{T}}
\end{aligned}
$$

169 For a membrane consisting of orthotropic layers, one can obtain the transformed material stiffness matrix $170\left[\bar{Q}^{(i)}\right]$ for the $i$ th lamina from its principal material stiffness matrix $\left[Q^{(i)}\right]$ and its ply angle (measured with 
171 respect to the axis $x$ ) by using tensor transformations and assuming that Jaumann stress $J_{33}^{(i)}=0$. The stress-strain relation for the ith lamina is given by

$$
\left\{J^{(i)}\right\}=\left[\bar{Q}^{(i)}\right]\{B\}
$$

176 where

$$
\left\{J^{(i)}\right\} \equiv\left\{J_{11}^{(i)}, J_{22}^{(i)}, J_{12}^{(i)}\right\}^{\mathrm{T}}, \quad\left[\bar{Q}^{(i)}\right] \equiv\left[\begin{array}{lll}
\bar{Q}_{11}^{(i)} & \bar{Q}_{12}^{(i)} & \bar{Q}_{16}^{(i)} \\
\bar{Q}_{12}^{(i)} & \bar{Q}_{22}^{(i)} & \bar{Q}_{26}^{(i)} \\
\bar{Q}_{16}^{(i)} & \bar{Q}_{26}^{(i)} & \bar{Q}_{66}^{(i)}
\end{array}\right]
$$

Using (10), (13) and (17) in (16) one can show that

$$
\delta \Pi=\sum_{i=1}^{N} \int_{A} \int_{z_{i}}^{z_{i+1}}\left(J_{11}^{(i)} \delta B_{11}+J_{22}^{(i)} \delta B_{22}+2 J_{12}^{(i)} \delta B_{12}\right) \mathrm{d} A \mathrm{~d} z=\sum_{i=1}^{N} \int_{A} \int_{z_{i}}^{z_{i+1}}\{\delta B\}^{\mathrm{T}}\left[\bar{Q}^{(i)}\right]\{B\} \mathrm{d} A \mathrm{~d} z
$$

204 where $A$ denotes the undeformed area of the reference surface, $N$ is the total number of layers, and $z_{i}$ and $205 z_{i+1}$ indicate the locations of the bottom and upper surfaces of the $i$ th layer. Substituting (11) into (18) 206 yields

$$
\delta \Pi=\int_{A}\{\delta \psi\}^{\mathrm{T}}[\Phi]\{\psi\} \mathrm{d} A
$$

210 where $[\Phi]$ is a symmetric $3 \times 3$ matrix given by 


$$
[\Phi]=\sum_{i=1}^{N} \int_{z_{i}}^{z_{i+1}}\left[\bar{Q}^{(i)}\right] \mathrm{d} z
$$

213 It follows from (12) and (1)-(4) that

$$
\{\delta \psi\}=[\Psi]\{\delta U\}
$$

217 where

$$
\begin{aligned}
& \{U\}=\left\{u, u_{x}, u_{y}, v, v_{x}, v_{y}, w, w_{x}, w_{y}\right\}^{\mathbf{T}} \\
& \Psi_{i j}=\frac{\partial \psi_{i}}{\partial U_{j}}
\end{aligned}
$$

223 The non-zero elements of $[\Psi]$ are:

$$
\begin{aligned}
& \Psi_{12}=C_{11} / C_{0}, \quad \Psi_{15}=C_{12} / C_{0}, \quad \Psi_{18}=C_{13} / C_{0}, \\
& \Psi_{13}=C_{14} / C_{0}, \quad \Psi_{18}=C_{15} / C_{0}, \quad \Psi_{19}=C_{16} / C_{0}, \\
& \Psi_{11}=\left(C_{12} k_{5}^{0}-C_{13} k_{1}^{0}+C_{15} k_{4}^{0}-C_{16} k_{62}^{0}\right) / C_{0} \\
& \Psi_{14}=-\left(C_{11} k_{5}^{0}+C_{13} k_{61}^{0}+C_{14} k_{4}^{0}+C_{16} k_{2}^{0}\right) / C_{0} \\
& \Psi_{17}=\left(C_{11} k_{1}^{0}+C_{12} k_{61}^{0}+C_{14} k_{62}^{0}+C_{15} k_{2}^{0}\right) / C_{0} \\
& \Psi_{22}=C_{21} / C_{0}, \quad \Psi_{25}=C_{22} / C_{0}, \quad \Psi_{28}=C_{23} / C_{0}, \\
& \Psi_{23}=C_{24} / C_{0}, \quad \Psi_{26}=C_{25} / C_{0}, \quad \Psi_{29}=C_{26} / C_{0}, \\
& \Psi_{21}=\left(C_{22} k_{5}^{0}-C_{23} k_{1}^{0}+C_{25} k_{4}^{0}-C_{26} k_{62}^{0}\right) / C_{0} \\
& \Psi_{24}=-\left(C_{21} k_{5}^{0}+C_{23} k_{61}^{0}+C_{24} k_{4}^{0}+C_{26} k_{2}^{0}\right) / C_{0} \\
& \Psi_{27}=\left(C_{21} k_{1}^{0}+C_{22} k_{61}^{0}+C_{24} k_{62}^{0}+C_{25} k_{2}^{0}\right) / C_{0} \\
& \Psi_{32}=2 C_{31} / C_{0}, \quad \Psi_{35}=2 C_{32} / C_{0}, \quad \Psi_{38}=2 C_{33} / C_{0}, \\
& \Psi_{33}=2 C_{34} / C_{0}, \quad \Psi_{36}=2 C_{35} / C_{0}, \quad \Psi_{39}=2 C_{36} / C_{0}, \\
& \Psi_{31}=2\left(C_{32} k_{5}^{0}-C_{33} k_{1}^{0}+C_{35} k_{4}^{0}-C_{36} k_{62}^{0}\right) / C_{0} \\
& \Psi_{34}=-2\left(C_{31} k_{5}^{0}+C_{33} k_{61}^{0}+C_{34} k_{4}^{0}+C_{36} k_{2}^{0}\right) / C_{0} \\
& \Psi_{37}=2\left(C_{31} k_{1}^{0}+C_{32} k_{61}^{0}+C_{34} k_{62}^{0}+C_{35} k_{2}^{0}\right) / C_{0}
\end{aligned}
$$

230 where

$$
\begin{aligned}
& C_{0} \equiv\left(1+e_{1}\right) \cos \gamma_{61}+\left(1+e_{2}\right) \cos \gamma_{62} \\
& C_{01} \equiv \frac{\widehat{T}_{21}-\sin \gamma_{6} \widehat{T}_{11}}{\cos \gamma_{6}}, \quad C_{02} \equiv \frac{\widehat{T}_{22}-\sin \gamma_{6} \widehat{T}_{12}}{\cos \gamma_{6}}, \quad C_{03} \equiv \frac{\widehat{T}_{23}-\sin \gamma_{6} \widehat{T}_{13}}{\cos \gamma_{6}} \\
& C_{04} \equiv \frac{\widehat{T}_{11}-\sin \gamma_{6} \widehat{T}_{21}}{\cos \gamma_{6}}, \quad C_{05} \equiv \frac{\widehat{T}_{12}-\sin \gamma_{6} \widehat{T}_{22}}{\cos \gamma_{6}}, \quad C_{06} \equiv \frac{\widehat{T}_{13}-\sin \gamma_{6} \widehat{T}_{23}}{\cos \gamma_{6}}
\end{aligned}
$$


$C_{14} \equiv-\left(1+e_{1}\right) \sin \gamma_{61} \sin \gamma_{62} \widehat{T}_{21}-\left(1+e_{1}\right) \sin \gamma_{61} \cos \gamma_{62} C_{04}$

$C_{15} \equiv-\left(1+e_{1}\right) \sin \gamma_{61} \sin \gamma_{62} \widehat{T}_{22}-\left(1+e_{1}\right) \sin \gamma_{61} \cos \gamma_{62} C_{05}$

$C_{16} \equiv-\left(1+e_{1}\right) \sin \gamma_{61} \sin \gamma_{62} \widehat{T}_{23}-\left(1+e_{1}\right) \sin \gamma_{61} \cos \gamma_{62} C_{06}$

$C_{21} \equiv-\left(1+e_{2}\right) \sin \gamma_{61} \sin \gamma_{62} \widehat{T}_{11}-\left(1+e_{2}\right) \sin \gamma_{62} \cos \gamma_{61} C_{01}$

$C_{22} \equiv-\left(1+e_{2}\right) \sin \gamma_{61} \sin \gamma_{62} \widehat{T}_{12}-\left(1+e_{2}\right) \sin \gamma_{62} \cos \gamma_{61} C_{02}$

$C_{23} \equiv-\left(1+e_{2}\right) \sin \gamma_{61} \sin \gamma_{62} \widehat{T}_{13}-\left(1+e_{2}\right) \sin \gamma_{62} \cos \gamma_{61} C_{03}$

$C_{24} \equiv\left(1+e_{2}+\left(1+e_{1}\right) \cos \gamma_{62} \cos \gamma_{61}\right) \widehat{T}_{21}-\left(1+e_{1}\right) \sin \gamma_{62} \cos \gamma_{61} C_{04}$

$C_{25} \equiv\left(1+e_{2}+\left(1+e_{1}\right) \cos \gamma_{62} \cos \gamma_{61}\right) \widehat{T}_{22}-\left(1+e_{1}\right) \sin \gamma_{62} \cos \gamma_{61} C_{05}$

$C_{26} \equiv\left(1+e_{2}+\left(1+e_{1}\right) \cos \gamma_{62} \cos \gamma_{61}\right) \widehat{T}_{23}-\left(1+e_{1}\right) \sin \gamma_{62} \cos \gamma_{61} C_{06}$

$C_{31} \equiv\left(1+e_{2}\right) \sin \gamma_{61} \cos \gamma_{62} \widehat{T}_{11}+\left(1+e_{2}\right) \cos \gamma_{61} \cos \gamma_{62} C_{01}$

$C_{32} \equiv\left(1+e_{2}\right) \sin \gamma_{61} \cos \gamma_{62} \widehat{T}_{12}+\left(1+e_{2}\right) \cos \gamma_{61} \cos \gamma_{62} C_{02}$

$C_{33} \equiv\left(1+e_{2}\right) \sin \gamma_{61} \cos \gamma_{62} \widehat{T}_{13}+\left(1+e_{2}\right) \cos \gamma_{61} \cos \gamma_{62} C_{03}$

$C_{34} \equiv\left(1+e_{1}\right) \cos \gamma_{61} \sin \gamma_{62} \widehat{T}_{21}+\left(1+e_{1}\right) \cos \gamma_{61} \cos \gamma_{62} C_{04}$

$C_{35} \equiv\left(1+e_{1}\right) \cos \gamma_{61} \sin \gamma_{62} \widehat{T}_{22}+\left(1+e_{1}\right) \cos \gamma_{61} \cos \gamma_{62} C_{05}$

$C_{36} \equiv\left(1+e_{1}\right) \cos \gamma_{61} \sin \gamma_{62} \widehat{T}_{23}+\left(1+e_{1}\right) \cos \gamma_{61} \cos \gamma_{62} C_{06}$

239 The way the components of $\{U\}$ are approximated defines a specific finite element. Using the finite-element discretization scheme, one can discretize the displacements as

$$
\{u, v, w\}^{\mathrm{T}}=[N]\left\{q^{(j)}\right\}
$$

244 where $\left\{q^{[j]}\right\}$ is the displacement vector of the $j$ th element and $[N]$ is a matrix of two-dimensional shape functions. For example, if it is an eight-node serendipity membrane element, we have

$$
\left\{q^{[j]}\right\}=\left\{\left\{q^{(k)}\right\}^{\mathrm{T}},\left\{q^{(k+1)}\right\}^{\mathrm{T}}, \ldots,\left\{q^{(k+7)}\right\}^{\mathrm{T}}\right\}^{\mathrm{T}}
$$

248 where $\left\{q^{(k)}\right\}$ is the displacement vector of the $k$ th node and is given by

$$
\left\{q^{(k)}\right\}=\left\{u^{(k)}, v^{(k)}, w^{(k)}\right\}^{\mathrm{T}}
$$

251 Substituting (30) into (22a) yields

$$
\{U\}=[D]\left\{q^{[j]}\right\}, \quad[D] \equiv[\partial][N]
$$

255 where $[\partial]$ is a $9 \times 3$ matrix consisting of differential operators and $[D]$ is a $9 \times 24$ matrix. Substituting (21) 256 and (33) into (19) yields

$$
\delta \Pi=\sum_{j=1}^{N_{\varepsilon}} \int_{A^{[j]}}\left\{\delta q^{[j]}\right\}^{\mathrm{T}}[D]^{\mathrm{T}}[\Psi]^{\mathrm{T}}[\Phi][\psi] \mathrm{d} A=\sum_{j=1}^{N_{\varepsilon}}\left\{\delta q^{[j]}\right\}^{\mathrm{T}}\left[K^{[j]}\right]\left\{q^{[j]}\right\}=\{\delta q\}^{\mathrm{T}}[K]\{q]
$$


where

$$
\left[K^{[j]}\right]\left\{q^{[j]}\right\} \equiv \int_{A^{[b]}}[D]^{\mathrm{T}}[\Psi]^{\mathrm{T}}[\Phi]\{\psi\} \mathrm{d} A
$$

$264 N_{\mathrm{e}}$ is the total number of elements, $A^{[j]}$ is the area of the $j$ th element, $\left[K^{[j]}\right]$ is the stiffness matrix of the $j$ th 265 element, $[K]$ is the structural stiffness matrix, and $\{q\}$ is the structural displacement vector. We note that $266\left[K^{[j]}\right]$ and $[K]$ are not explicitly written.

Because the structural stiffness matrix is a non-linear function of displacements, the governing equations need to be solved by an incremental/iterative method. To derive incremental equations, we let

$$
\left\{q^{(j)}\right\}=\left\{q^{0}\right\}+\left\{\Delta q^{(j)}\right\}, \quad\{U\}=\left\{U^{0}\right\}+\{\Delta U\}
$$

271 where $\left\{q^{0}\right\}$ denotes the equilibrium solution and $\left\{\Delta q^{[j]}\right\}$ the incremental displacement vector.

272 Because $\{J\}=[\bar{Q}]\{\psi\}$ and initial strains (or stresses) will be considered in the formulation, we will replace $273\{\psi\}$ with

$$
\{\psi\} \rightarrow\left\{\psi_{0}\right\}+\{\psi\}
$$

276 where $\left\{\psi_{0}\right\}$ represent known initial strains in the initial configuration described by the $x y z$ coordinate sys-

277 tem, and $\{\psi\}$ represent unknown additional strains caused by additional loads. Hence, we obtain the first278 order expansions of $\{\psi\}$ and $[\Psi]$ as

$$
\{\psi\}=\left\{\psi_{0}\right\}+\left\{\psi^{0}\right\}+\left[\Psi^{0}\right]\{\Delta U\}
$$

282 and

$$
[\Psi]=\left[\Psi^{0}\right]+[\Xi]
$$

286 where the entry $\Xi_{i j}$ of $[\Xi]$ is given by

$$
\Xi_{i j}=\frac{\partial^{2} \psi_{i}}{\partial U_{j} \partial U_{k}} \Delta U_{k}
$$

290 Then, we use (38) and (39) to expand (35) into a Taylor series and neglect higher-order terms to obtain

$$
\left[K^{[j]}\right]\left\{q^{[j]}\right\}=\int_{A^{v i}}\left[[D]^{\mathrm{T}}\left[\Psi^{0}\right]^{\mathrm{T}}[\Phi]\left(\left\{\psi_{0}\right\}+\left\{\psi^{0}\right\}\right)+[D]^{\mathrm{T}}\left[\Psi^{0}\right]^{\mathrm{T}}[\Phi]\left[\Psi^{0}\right]\{\Delta U\}+[D]^{\mathrm{T}}[\Xi]^{\mathrm{T}}[\Phi]\left(\left\{\psi_{0}\right\}+\left\{\psi^{0}\right\}\right)\right] \mathrm{d} A
$$

294 Using (40) and direct expansion, one can prove that

$$
\begin{aligned}
{[\Xi]^{\mathrm{T}}[\Phi]\left(\left\{\psi_{0}\right\}+\left\{\psi^{0}\right\}\right) } & =\left\{\Xi_{m i} \Phi_{m n}\left(\psi_{0 n}+\psi_{n}^{0}\right)\right\}=\left\{\frac{\partial \Psi_{m i}}{\partial U_{j}} \Delta U_{j} \Phi_{m n}\left(\psi_{0 n}+\psi_{n}^{0}\right)\right\} \\
& =\left\{\frac{\partial^{2} \psi_{m}^{0}}{\partial U_{i} \partial U_{j}} \Phi_{m n}\left(\psi_{0 n}+\psi_{n}^{0}\right) \Delta U_{j}\right\} \\
& =[\Omega]\{\Delta U\}
\end{aligned}
$$

298 where $[\Omega]$ is a symmetric matrix and its entry $\Omega_{i j}$ is given by

$$
\Omega_{i j}=\frac{\partial^{2} \psi_{m}^{0}}{\partial U_{i} \partial U_{j}} \Phi_{m n}\left(\psi_{0 n}+\psi_{n}^{0}\right)=\frac{\partial^{2} \psi_{m}^{0}}{\partial U_{j} \partial U_{i}} \Phi_{m n}\left(\psi_{0 n}+\psi_{n}^{0}\right)=\Omega_{j i}
$$

301 Hence, substituting (42) and (33) into (41) yields 


$$
\left[K^{[j]}\right]\left\{q^{[j]}\right\}=\left[K^{[j]}\right]\left\{\Delta q^{[j]}\right\}+\left.\left[K^{[j]}\right]\left\{q^{[j]}\right\}\right|_{\left\{q^{[j]}\right\}=\left\{q^{0}\right\}}
$$

304 where $\left[K^{-[i]}\right]$ is the so-called elemental tangent stiffness matrix and is given by

$$
\left[\breve{K}^{\left[{ }^{[j]}\right.}\right]=\int_{A^{(j)}}[D]^{\mathrm{T}}\left(\left[\Psi^{0}\right]^{\mathrm{T}}[\Phi]\left[\Psi^{0}\right]+[\Omega]\right)[D] \mathrm{d} A
$$

308 and

$$
\left.\left[K^{[j]}\right]\left\{q^{[j]}\right\}\right|_{\left\{q^{(j)}\right\}=\left\{q^{0}\right\}}=\int_{A^{[j]}}[D]^{\mathrm{T}}\left[\Psi^{0}\right]^{\mathrm{T}}[\Phi]\left(\left\{\psi_{0}\right\}+\left\{\psi^{0}\right\}\right) \mathrm{d} A
$$

311 We note that $\left[K^{-b^{\prime}}\right]$ is symmetric. Eq. (41) shows that initial strains and additional equilibrium strains play

$$
[K]\{q\}=\lambda\left\{R_{r}\right\}
$$

$$
\begin{aligned}
\delta W_{\mathrm{nc}} & =\int_{A}\left(r_{1} \delta u+r_{2} \delta v+r_{3} \delta w\right) \mathrm{d} A=\int_{A}\left\{\begin{array}{llll}
\delta u & \delta v & \delta w
\end{array}\right\}\left\{\begin{array}{lll}
r_{1} & r_{2} & r_{3}
\end{array}\right\}^{\mathrm{T}} \mathrm{d} A \\
& =\sum_{j=1}^{N_{\mathrm{c}}}\left\{\delta q^{[j]}\right\}^{\mathrm{T}}\left\{R^{[j]}\right\}=\{\delta q\}^{\mathrm{T}}\{R\}
\end{aligned}
$$

where $r_{1}, r_{2}$, and $r_{3}$ are distributed external loads per unit area along the axes $x, y$ and $z,(30)$ has been used, $\left\{R^{[j}\right\}$ is the elemental nodal loading vector, $\{R\}$ is the structural nodal loading vector, and

$$
\left\{R^{[U]}\right\} \equiv \int_{A}[N]^{\mathrm{T}}\left\{\begin{array}{lll}
r_{1} & r_{2} & r_{3}
\end{array}\right\}^{\mathrm{T}} \mathrm{d} A
$$

\subsection{Incremental-iterative solution method}

With the use of a loading parameter $\lambda$, the governing equation of a static problem can be obtained from $(34),(47)(15)$ as

where $\left\{R_{r}\right\}(=\{R\} / \lambda)$ is a reference load vector. Let

$$
\begin{aligned}
& \{q\} \equiv\{q\}_{i}=\left\{q^{0}\right\}+\{\Delta q\}_{i}=\{q\}_{i-1}+\{\delta q\}_{i} \\
& \lambda \equiv \lambda_{i}=\lambda^{0}+\Delta \lambda_{i}=\lambda_{i-1}+\delta \lambda_{i}
\end{aligned}
$$

338 where $i \geqslant 2, i$ is the number of iterations in searching for a converged solution when the load increases from $339 \lambda=\lambda^{0}$ to $\lambda=\lambda^{0}+\Delta \lambda_{i},\left\{q^{0}\right\}$ denotes the equilibrium solution corresponding to $\lambda=\lambda^{0},\{q\}_{i}$ denotes the iterated solution corresponding to $\lambda=\lambda_{i}$, and $\{\Delta q\}_{i}$ denotes the incremental displacement vector correspond-

\footnotetext{
2 results into a Taylor series, and neglecting higher-order terms, we obtain
} 


$$
[\widehat{K}]_{i-1}\{\delta q\}_{i}=\delta \lambda_{i}\left\{R_{r}\right\}_{i-1}+\{g\}_{i-1}
$$

346 where

$$
\begin{aligned}
& \{g\}_{i-1} \equiv \lambda_{i-1}\left\{R_{r}\right\}_{i-1}-([K]\{q\})_{i-1},\left\{R_{r}\right\}=\left\{R_{r}\right\}_{i-1}+[\bar{K}]_{i-1}\{\delta q\}_{i} \\
& {[\widehat{K}]_{i-1} \equiv[\widetilde{K}]_{i-1}-\lambda_{i-1}[\bar{K}]_{i-1}}
\end{aligned}
$$

349 Here $[\bar{K}]$ is a non-trivial matrix only if parametric loadings exist, $[\widehat{K}]_{i-1}$ is the local tangent stiffness matrix, 350 and the sub-indices indicate the state, e.g., $[\widehat{K}]_{i-1} \equiv[\widehat{K}]_{\{q\}=\{q\}_{i-1}, \lambda=\lambda_{i-1}}$. To solve for $\{\delta q\}_{i}$ and $\delta \lambda_{i}$ we use an

351 alternative version of Riks' method (Riks, 1979; Lee, 1992) to solve (51) and the following constraint 352 equation

$$
\{\Delta q\}_{i-1}^{\mathrm{T}}\{\delta q\}_{i}+\Delta \lambda_{i-1} \delta \lambda_{i}\left\{R_{r}\right\}_{i-1}^{\mathrm{T}}\left\{R_{r}\right\}_{i-1}=0
$$

which limits the arc-length increment by confining the current search direction to be perpendicular to the previous accumulated searching direction (Riks, 1979).

\subsection{Modal analysis}

After the statically deformed geometry of a membrane is obtained using the iteration method shown above, the tangent stiffness matrix $[K]$ (see (45)) of the final deformed state is the stiffness matrix for the modal analysis. The mass matrix $[M]$ needed for modal analysis is derived through the variation of kinetic energy $\delta T$ by using (30) as

$$
\begin{aligned}
\delta T & =-\int_{A} \int_{z} \rho(\ddot{u} \delta u+\ddot{v} \delta v+\ddot{w} \delta w) \mathrm{d} A \mathrm{~d} z=-\sum_{j=1}^{N_{\mathrm{e}}} \int_{A^{(j]}}\left\{\delta q^{[j]}\right\}^{\mathrm{T}}[N]^{\mathrm{T}} m[N]\left\{\ddot{q}^{[j]}\right\} \mathrm{d} A \\
& =-\sum_{j=1}^{N_{e}}\left\{\delta q^{[j]}\right\}^{\mathrm{T}}\left[M^{[j]}\right]\left\{\ddot{q}^{[j]}\right\}=-\{\delta q\}^{\mathrm{T}}[M]\{\ddot{q}\}
\end{aligned}
$$

where $\rho$ is the mass density, $\left[M^{[0]}\right]$ is the elemental mass matrix, $[M]$ is the structural mass matrix, and

$$
m=\int_{z} \rho \mathrm{d} z, \quad\left[M^{\mathrm{b}}\right] \equiv \int_{A}[N]^{\mathrm{T}} m[N] \mathrm{d} A
$$

67 The elemental tangent mass matrix is the same as $\left[M^{[j]}\right]$ because it is a constant matrix.

\section{Numerical results}

\subsection{Inflated circular cylindrical tube}

We consider a membrane tube having a diameter $D=3 \mathrm{in}$. and a length $L=47.5 \mathrm{in}$. with two ends fixed. The tube was made by overlapping (by $0.5 \mathrm{in}$.) and gluing two opposite edges of a rectangular Kapton film using a $0.001^{\prime \prime}$ thick double-sided Kapton tape with silicone adhesive. According to the manufacturer the Kapton film has Young's modulus $E=3.7 \times 10^{5} \mathrm{psi}$, mass density $\rho=2.7552$ slugs $/ \mathrm{ft}^{3}$, thickness $h=0.002$ in., and Poisson's ratio $v=0.34$. The seam is assumed to have a width $w=0.5 \mathrm{in}$. and thickness $h=0.005$ in. In numerical analyses we focus on the effects of the longitudinal joining seam and different pressures on mode shapes and natural frequencies of a pressurized tube.

Fig. 2 shows the tube modeled using rectangular membrane elements with the same thickness, where elements are shrunk by $20 \%$ in order to show the connectivity of elements. Fig. 3 shows a single point Fre- 

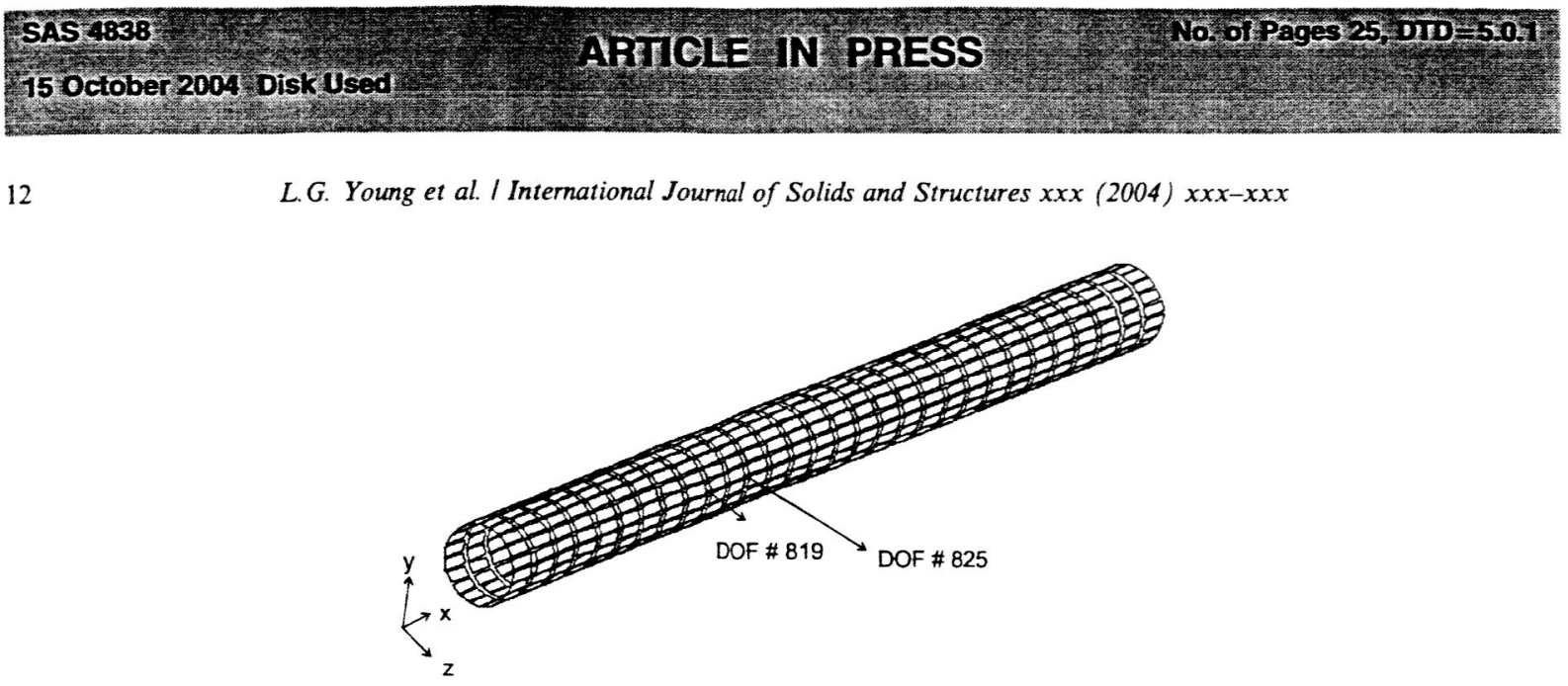

Fig. 2. The inflated circular cylindrical tube modeled using rectangular membrane elements.

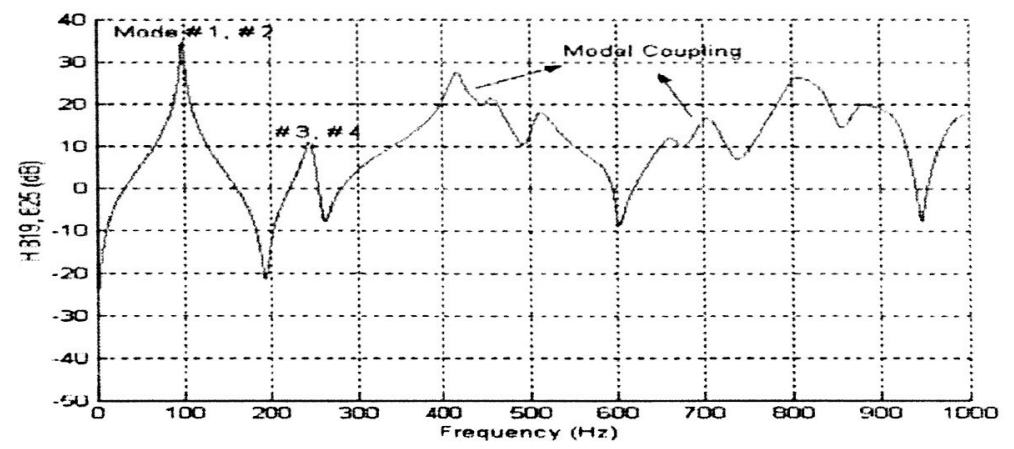

Fig. 3. A single point FRF of the inflated tube without a joining seam when $p=1.75$ psi, where the excitation is at DOF 825 and the response at DOF 819 in Fig. 2.

quency Response Function (FRF) with an excitation at DOF 825 (see Fig. 2, a nodal transverse displacement $w$ ) and the response at DOF 819 when the tube is inflated with $p=1.75$ psi. The FRF is obtained using the first 100 modes with modal damping ratios assumed to be 0.02 for each mode. We note that clear peaks only show at modes \#1 (\#2) and \#3 (\#4) and there are no clear peaks in high-frequency ranges because of modal coupling. Fig. 4 shows the first 12 mode shapes and corresponding natural frequencies of the tube inflated with $p=1.75$ psi. Because the thickness of the tube is uniform, mode shapes appear in pairs because of the symmetric geometry. Hence, modes \#1, \#3, \#5,\#7,\#9, and \#11 are the same as modes $\# 2, \# 4, \# 6, \# 8, \# 10$, and $\# 12$, respectively. We notice that only modes \#1, \#3, and \#9 are global bending modes and most of other modes are local shell modes. Table 1 shows that reducing the internal pressure from $p=1.75 \mathrm{psi}$ to $p=0.8125 \mathrm{psi}$ reduces the natural frequencies, and it also changes the appearance sequence of mode shapes after the eighth mode. For example, the 11th mode becomes the new 9 th mode, the new 11th mode is a global torsional mode that does not appear in pair, and the third bending mode becomes the new 15th (and 14th) mode. Table 1 also shows that, when $p$ decreases, the natural frequencies of global bending modes only reduce a little, but the ones of shell modes (e.g., modes \#5 and \#7) reduce dramatically.

Fig. 5 show the tube modeled using a thickness of $h=0.005 \mathrm{in}$. for elements on the seam and $h=0.002$ in. for other elements. The FRFs shown in Fig. 6 show again that there are no clear peaks except the two peaks in the low-frequency range caused by the bending modes. Because shell modes dominate high-frequency responses and their frequencies are close to each other, modal coupling is expected and it 
Mode \# 1, 2: $97.7 \mathrm{~Hz}$

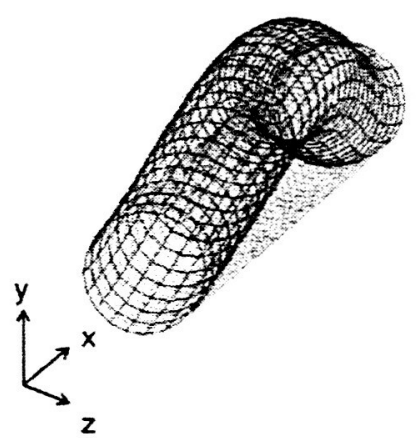

Mode \# 5, 6: $420.1 \mathrm{~Hz}$

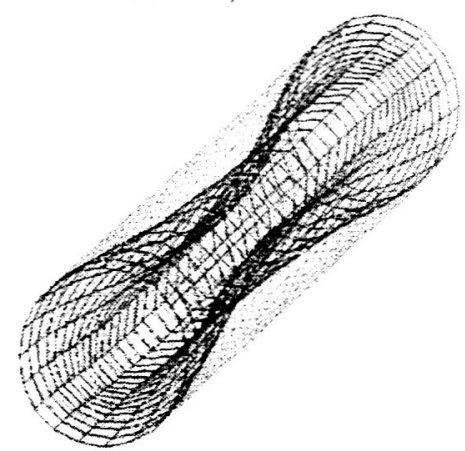

Mode \# 9, 10: $439.5 \mathrm{~Hz}$

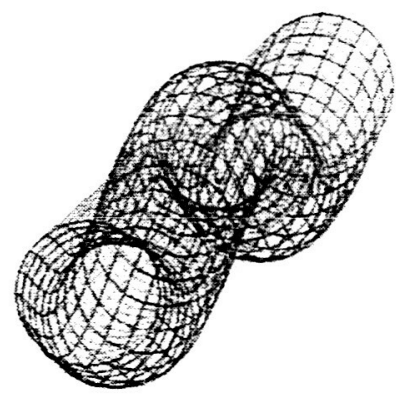

Mode \#, 4: $245.8 \mathrm{~Hz}$

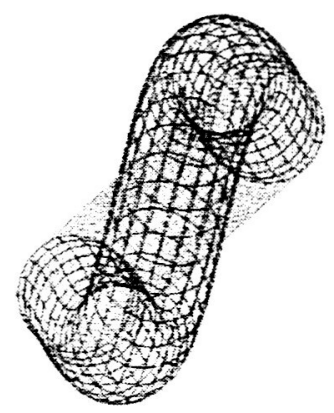

Mode \# 7, 8: $429.6 \mathrm{~Hz}$

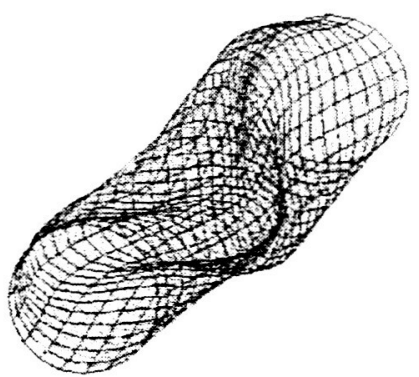

Mode \#11, 12: $456.8 \mathrm{~Hz}$

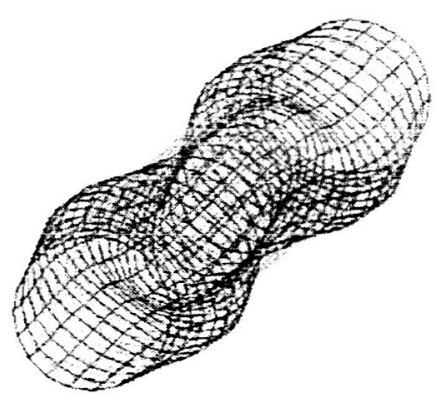

Fig. 4. The first 12 mode shapes and natural frequencies of the inflated tube without a joining seam and having an inflation pressure $p=1.75$ psi.

will be difficult in obtaining mode shapes in experiments. Fig. 7 shows four low-frequency mode shapes and natural frequencies when $p=1.75 \mathrm{psi}$. Because of the joining seam, the geometric symmetry is broken and

natural frequencies $\omega_{1}$ and $\omega_{3}$ are different from $\omega_{2}$ and $\omega_{4}$, respectively. However, the first four modes are still global bending modes, and shell modes still dominate high-frequency responses. We note that $\omega_{2}>\omega_{1}$ 2 because mode \#2 involves the bending of the joining seam, the $\omega_{2}$ is higher than the $\omega_{2}$ in Fig. 4, and the $\omega_{1}$ 403 is lower than the $\omega_{1}$ in Fig. 4. Modes \#2 and \#4 do not cause peaks in Fig. 6 because they are bending on 4 the $x y$ plane (see Fig. 5). 


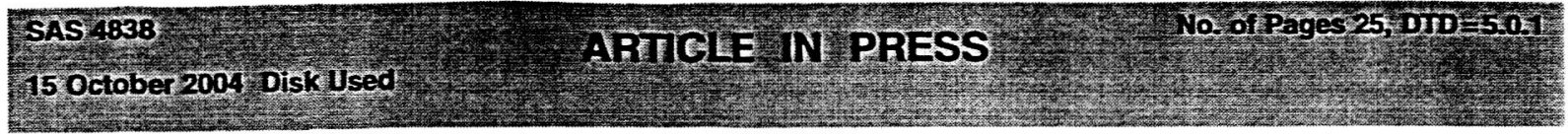

L.G. Young et al. I International Journal of Solids and Structures $x x x$ (2004) $x x x-x x x$

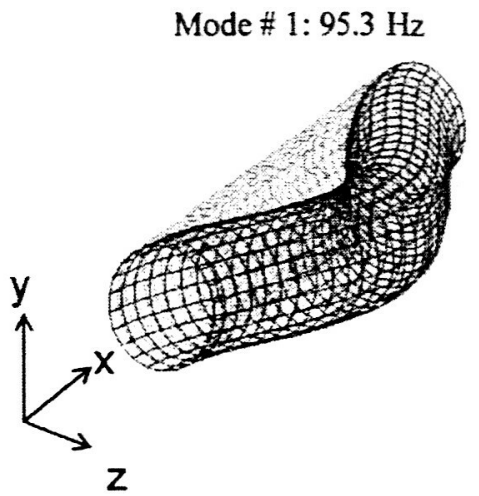

Mode \# 2: $99.2 \mathrm{~Hz}$

Mode \# 3: $240.6 \mathrm{~Hz}$

Mode \# 5: $398.9 \mathrm{~Hz}$
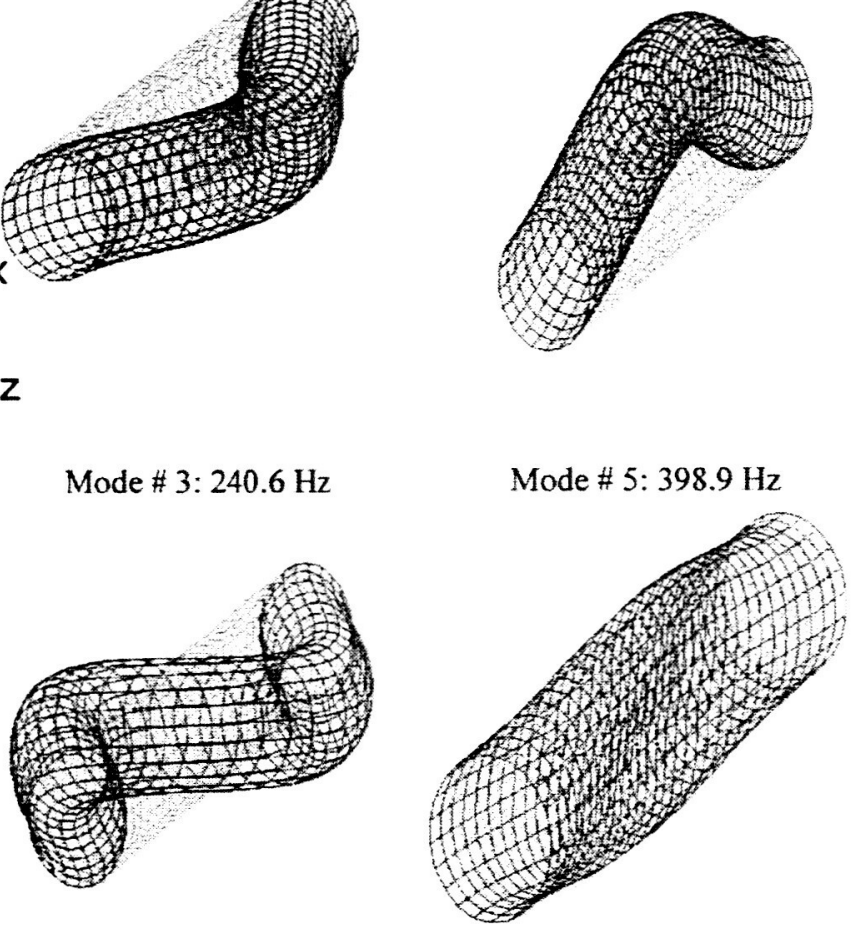

Fig. 7. Four low-frequency mode shapes and natural frequencies of the inflated tube with a joining seam and $p=1.75$ psi.

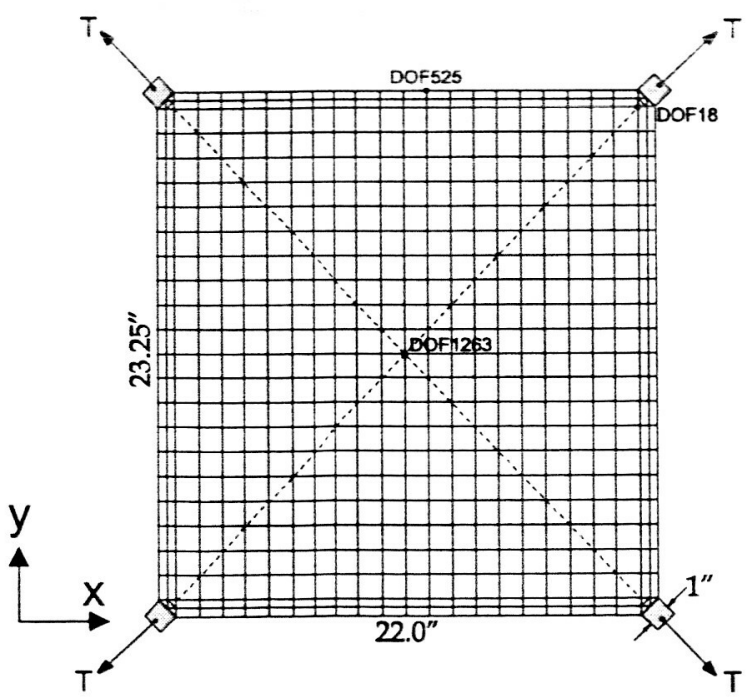

Fig. 8. The rectangular Kapton membrane with four corners loaded. 


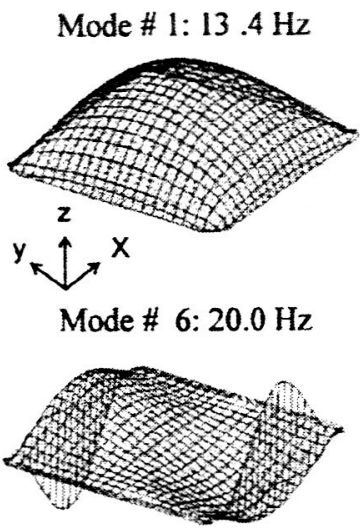

Mode \#10: $24.4 \mathrm{~Hz}$

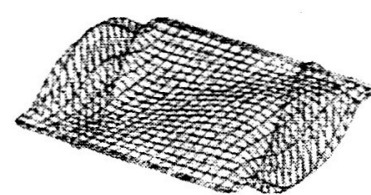

Mode \# 2: $17.7 \mathrm{~Hz}$

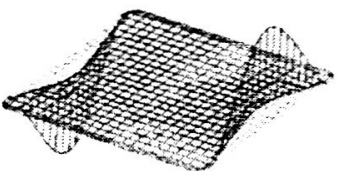

Mode \# 8: $21.19 \mathrm{~Hz}$

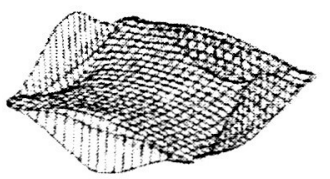

Mode \#11: $24.7 \mathrm{~Hz}$

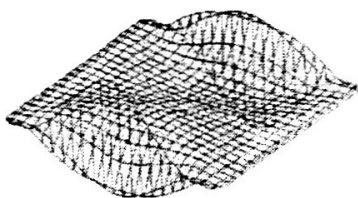

Mode \# 3: $17.7 \mathrm{~Hz}$

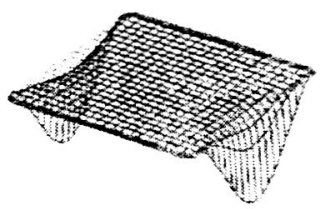

Mode \# 9: $22.6 \mathrm{~Hz}$

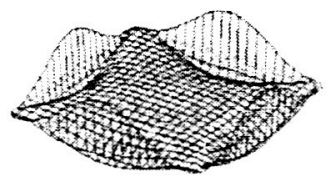

Mode \#12: $27.0 \mathrm{~Hz}$

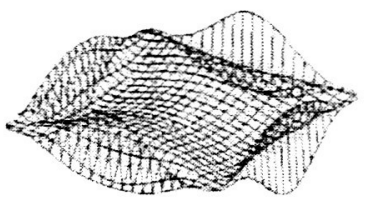

Fig. 9. Some low-frequency mode shapes and natural frequencies of the tensioned rectangular membrane with $T=1.5 \mathrm{lbs}$.

412 global modes. These local modes are due to the non-uniform tension field over the entire membrane with 413 the four edges being under small tension forces. For example, modes \#2 and \#3 are apparently local modes 414 and modes \#1, \#6, and \#9 are more like global modes. We note that modes \#2, \#3, \#6, and \#10 are dom415 inated by vibrations of the two edges parallel to the $x$ axis, and modes \#4, \#5, \#7, and \#11 are the corre416 sponding local modes dominated by vibrations of the two edges parallel to the $y$ axis. Because the 417 membrane shape is non-square, the tension force along the two edges parallel to the $y$ axis is higher than 418 that along the two edges parallel to the $x$ axis and hence the natural frequencies of modes \#4, \#5, \#7, and 419 \#11 are slightly higher than those of modes $\# 2, \# 3$, \#6, and $\# 10$, respectively. Table 2 compares the natural 420 frequencies when $T=2 \mathrm{lbs}$ with those when $T=1.5 \mathrm{lbs}$. We note that increasing the tension force by $0.5 \mathrm{lb}$ does not change the appearance sequence of the first few mode shapes, but the natural frequencies increase. Fig. 10a and b show FRFs of a response point at the center (DOF 1263, see Fig. 8) and a point close to the center of an edge (DOF 525), respectively. The FRFs are obtained using the first 70 modes with modal damping ratios assumed to be 0.02 for each mode. Fig. 9 shows that the dynamics around the edge is dominated by local modes and the dynamics around the membrane center is dominated by global modes, and Fig. 10a,b exactly show this phenomenon. Similar to those observed in the FRFs of the Kapton tube, the local modes have natural frequencies close to each other and hence modal coupling exists in all high-frequency ranges.

Table 2

Comparison of natural frequencies of the rectangular membrane when the tension force is changed from $T=1.5 \mathrm{lb}$ to $T=2 \mathrm{lb}$

\begin{tabular}{llllll}
\hline & $\omega_{1}(\mathrm{~Hz})$ & $\omega_{2}(\mathrm{~Hz})$ & $\omega_{3}(\mathrm{~Hz})$ & $\omega_{4}(\mathrm{~Hz})$ & $\omega_{5}(\mathrm{~Hz})$ \\
\hline$T=1.5 \mathrm{lb}$ & 13.4 & 17.7 & 17.7 & 18.5 & 18.6 \\
$T=2 \mathrm{lb}$ & 15.5 & 20.5 & 20.5 & 21.4 & 21.5 \\
\hline
\end{tabular}



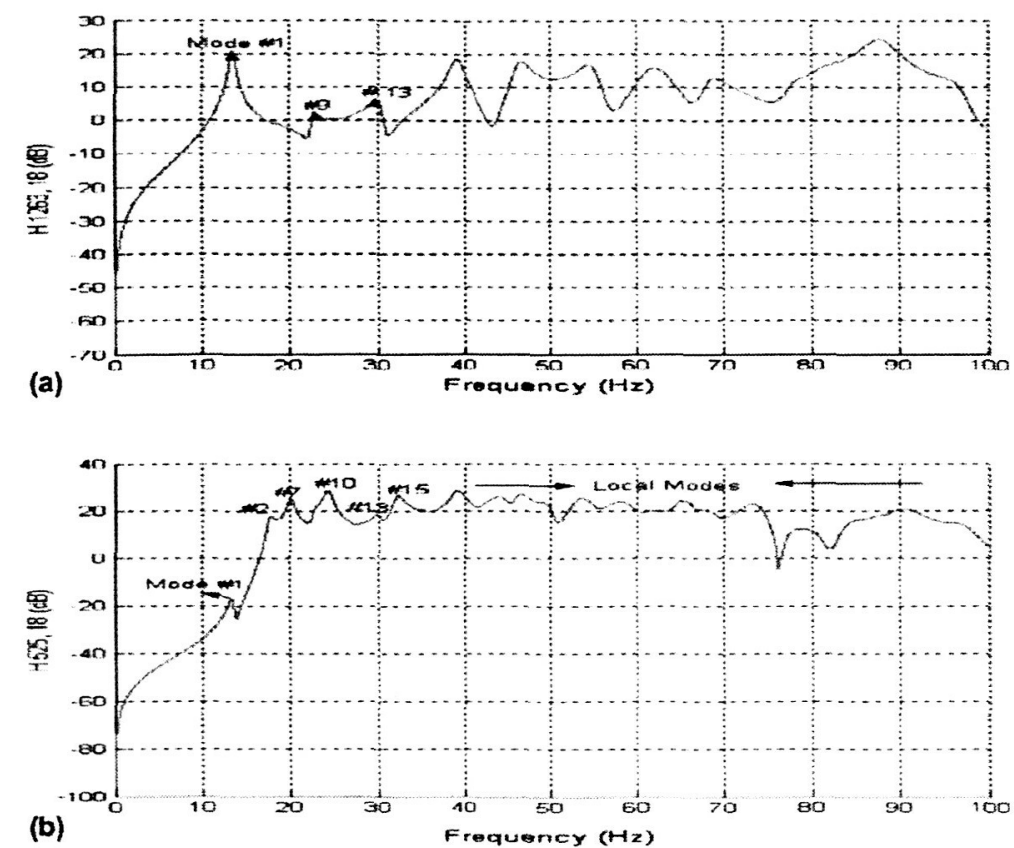

Fig. 10. Single point FRFs of the tensioned membrane when $T=1.5 \mathrm{lbs}$ : (a) measured at DOF 1263 in Fig. 8 , and (b) measured at DOF 525.

\section{5. Experimental results and comparison}

\section{5.1. Inflated circular cylindrical tube}

Fig. 11 shows the circular cylindrical Kapton tube set-up with 100 circular retro-refiective markers on it 432 and a Ling Dynamic LDS V408 shaker attached to the plastic part that sealed and supported the left end of the tube. Because the membrane is transparent, the retro-refiective markers were used to make the measurement using a Polytec PI PSV-200 scanning laser vibrometer possible and to enhance the laser signal. However, the markers are small $\left(\approx 0.06\right.$ in. $\left.^{2}\right)$ and mass loading from the markers is negligible. The retroreflective markers look big in Fig. 11 because they reflected the flashlight from the camera. The seam is on the opposite side of the 100 measurement markers. Experiments were performed for inflation pressures $438 p=0.8125 \mathrm{psi}$ and $p=1.75 \mathrm{psi}$. For the FFT acquisition in using the scanning laser vibrometer, a $0-1 \mathrm{kHz}$ periodic chirp excitation was used with 6400 FFT lines. Fig. 12a shows the averaged FRF of the tube with

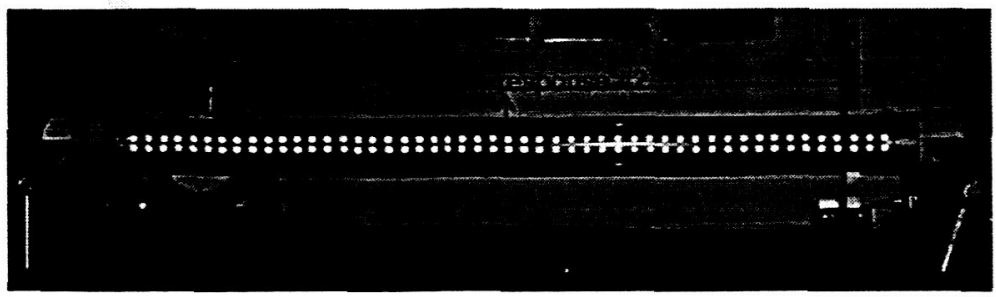

Fig. 11. The inflated circular cylindrical Kapton tube with retro-reflective circular markers. 

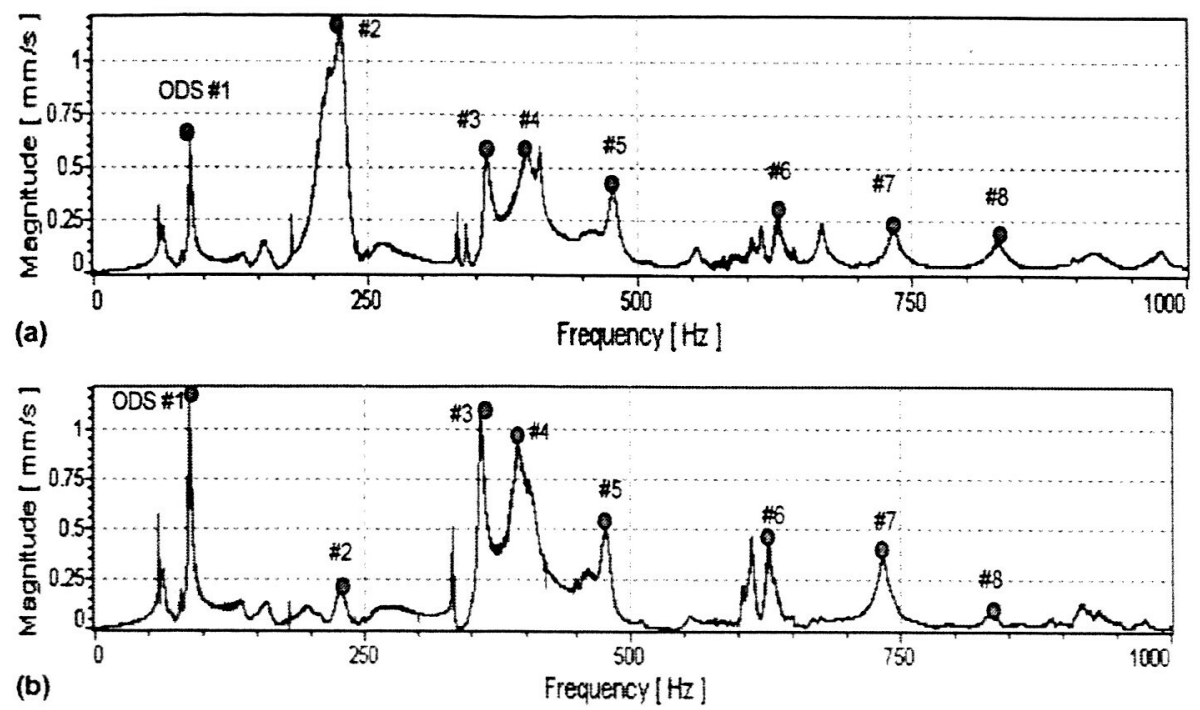

(b)

Frequency $[\mathrm{Hz}]$

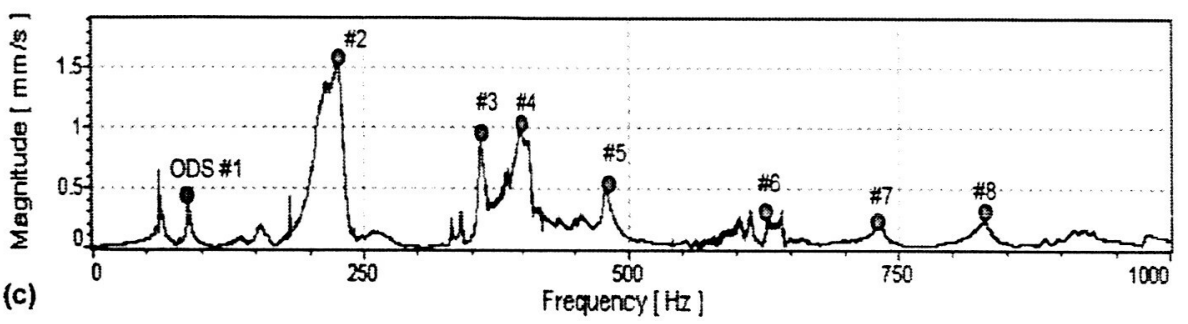

Fig. 12. The FRFs of the inflated tube with an inflation pressure $p=1.75$ psi: (a) averaged FRF, (b) FRF measured at center, and (c) FRF measured near the excitation location.

an inflation pressure $p=1.75$ psi. Fig. $12 \mathrm{~b}$ and $\mathrm{c}$ shows FRFs measured at the center (see Fig. 11, \#2) and near the excitation (\#1), respectively. We notice that the FRFs have many small peaks but the dominants peaks are in the low-frequency range, as expected. Moreover, because the membrane is so flexible, it was difficult to excite high-frequency modes and hence the peaks in high-frequency ranges have small amplitudes. The first six Operational Deflection Shapes (ODSs) corresponding to the peaks in Fig. 12 are shown in Fig. 13. The ODSs \#1 and \#2 in Fig. 13 correspond to and agree well with modes \#2 and \#4 in Fig. 7 . However, the experimental frequencies are lower than the numerical ones by $11.5 \mathrm{~Hz}(11.6 \%)$ and $20.6 \mathrm{~Hz}$ $(8.3 \%)$, respectively. The discrepancy could be the effect of air mass or due to incorrect material properties used in the numerical computation. Since Young's modulus of Kapton films is known to be a function of strains, temperature, and time, the value of $E$ used in the finite-element analysis may not be accurate. Air mass may significantly change the dynamic characteristics of membrane structures and needs to be accounted for in the numerical analysis in order to have results comparable to experimental ones (Kukathasan and Pellegrino, 2002).

We note that ODS \#4 corresponds to a traveling wave and thus is a coupled mode. The ODS corresponding to the peak at $60 \mathrm{~Hz}$ was found to be a non-uniform traveling shape, and hence it is not an ODS at all. It was believed to be due to the electric power used to drive the electro-mechanical shaker. The ODSs in Fig. 13 look like pure bending modes. However, because the measurement was along a line, it is difficult to know if a measured ODS is a bending mode or a shell mode. 


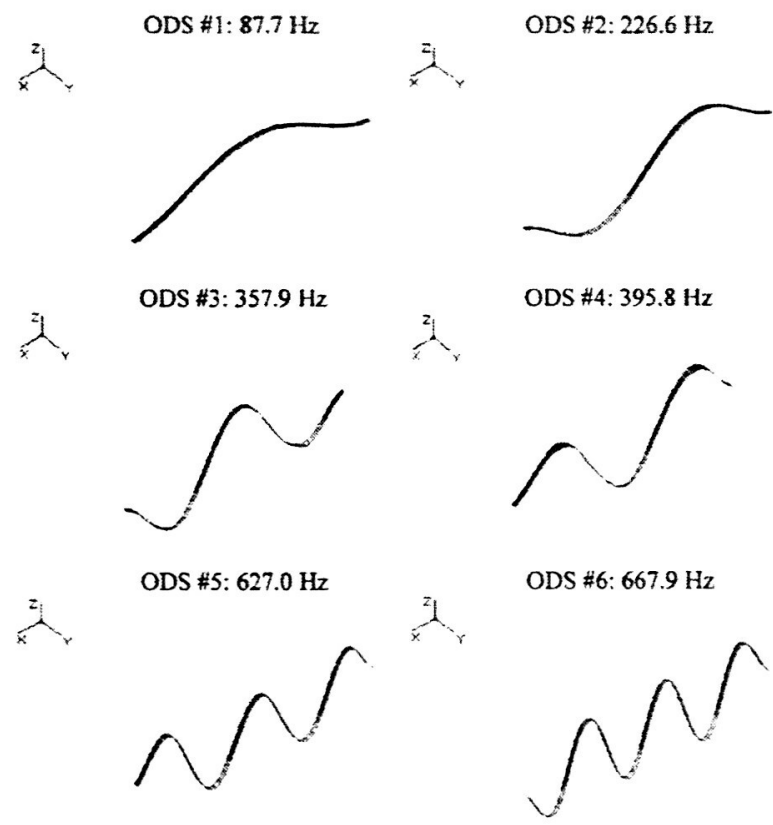

Fig. 13. The first six ODSs and natural frequencies of the inflated Kapton tube with a pressure $p=1.75 \mathrm{psi}$.

When the internal pressure is reduced from $p=1.75 \mathrm{psi}$ to $p=0.8125 \mathrm{psi}$, the frequency of ODS \#2 decreased from $226.6 \mathrm{~Hz}$ (see Fig. 13) to $211.4 \mathrm{~Hz}$.

\subsection{Tensioned rectangular membrane}

Fig. 14 shows the test set-up of the 22 in. $\times 23.25$ in. $\times 0.002$ in. rectangular membrane. Experiments were performed with an excitation at a single corner and with a simultaneous excitation at the four corners, respectively. The number of measurement points is $13 \times 16$, as shown in Fig. 14. The small circular retro-reflective markers have a radius of $0.14 \mathrm{in}$., but they look big in the picture because they reflected the camera flashlight. Because of the local flexibility of membranes, the excitation points were positioned at where the tension cables were connected to the membrane at the four corners. Fig. 15 shows the averaged FRF and a single-point FRF of the rectangular membrane with $T=1.5 \mathrm{lbf}$ and an excitation at the lower left corner. The peak at $60 \mathrm{~Hz}$ in Fig. $15 \mathrm{a}$ was caused by the $60-\mathrm{Hz}$ electrical power used to drive the shaker. The many small peaks in Fig. 15b around high-frequency ranges are due to local modes around the edges, as explained and shown in Figs. 9 and 10. Fig. 16 shows the first four ODSs and frequencies under the single point excitation. Fig. 17 shows the first four ODSs and frequencies obtained with $T=1.5 \mathrm{lbf}$ and a simultaneous excitation at the four corners. In order to simultaneously excite the four corners, the four corners were supported by a frame and a Z-shape rod was used to connect the frame to the shaker (see Fig. 14). The obtained FRFs are rougher than those in Fig. 15, which is probably due to the vibration of the rod.

Figs. 16 and 17 show that the excitation location may affect the natural frequencies and mode shapes. ODSs \#1 and \#4 in Fig. 16 correspond to ODSs \#1 and \#3 in Fig. 17, and ODS \#3 in Fig. 16 is similar to ODS \#2 in Fig. 17. The ODSs \#1, \#2, and \#3 in Fig. 17 correspond to and agree with the modes \#1, \#6, and \#9 in Fig. 9. However, the experimental frequencies are much lower than the numerical ones. Since the rectangular membrane has a large area in contact with the ambient air, air mass significantly lowers the experimental natural frequencies. Moreover, Fig. 15 shows that there are no clear peaks beyond $20 \mathrm{~Hz}$. 


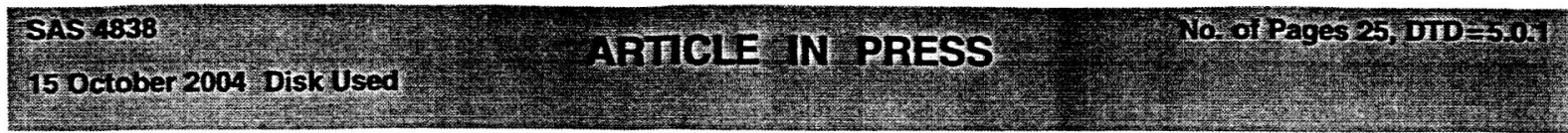

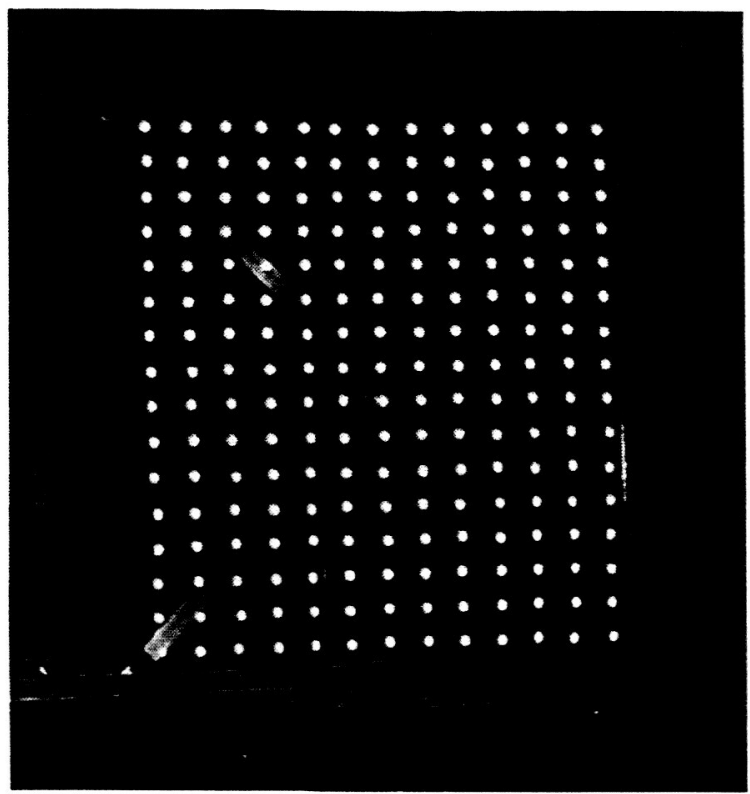

Fig. 14. The rectangular Kapton membrane excited at the four corners by a frame with a tension force $T=1.5 \mathrm{lbs}$ at each corner.
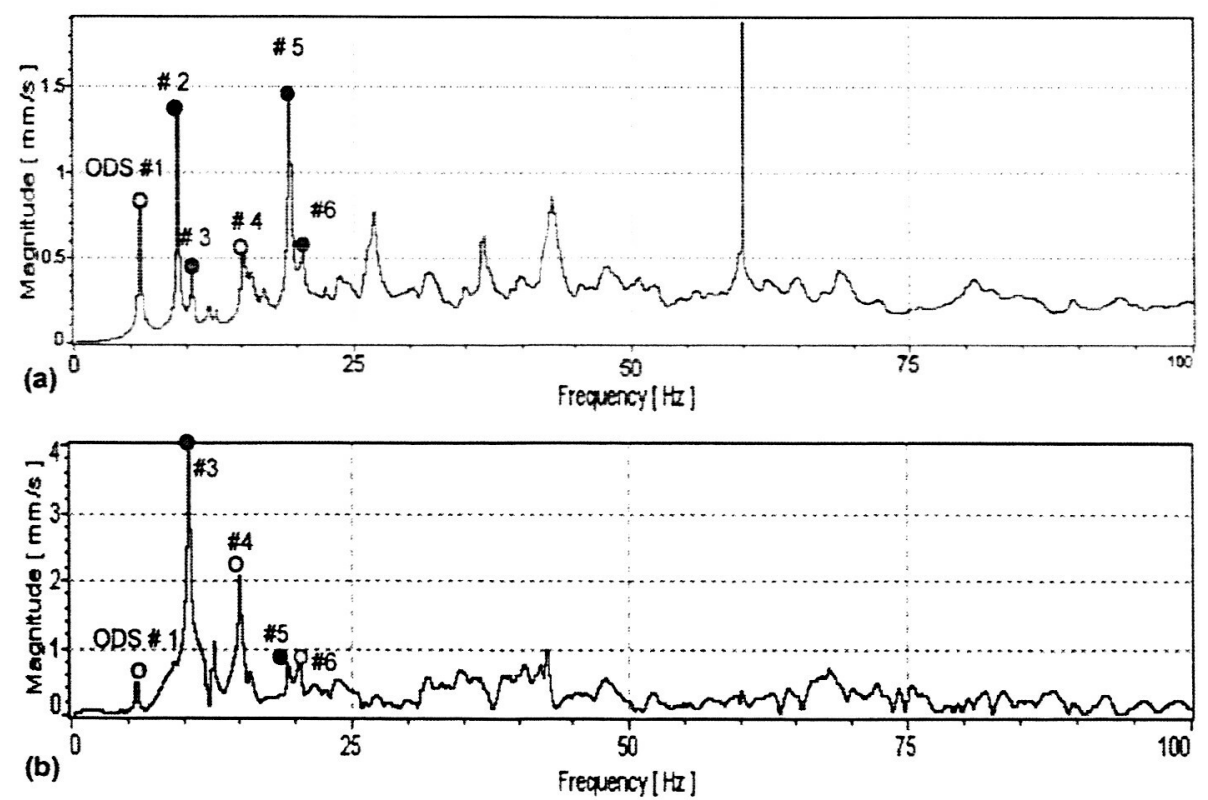

Fig. 15. FRFs of the rectangular membrane with $T=1.5 \mathrm{lbs}$ when the excitation is at the lower left corner: (a) averaged FRF, and (b) single point FRF measured at the center of the bottom edge.

481 In other words, high-frequency modes are highly coupled, and most experimental ODSs were observed to 482 be traveling modes due to modal couplings. Hence, it is difficult to obtain experimental high-frequency 


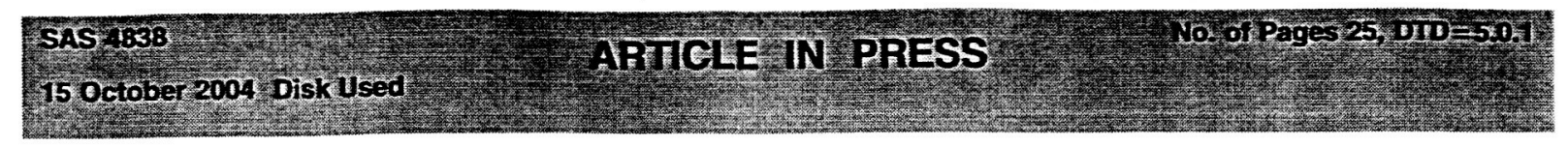

L.G. Young et al. I International Journal of Solids and Structures $x x x$ (2004) $x x x-x x x$
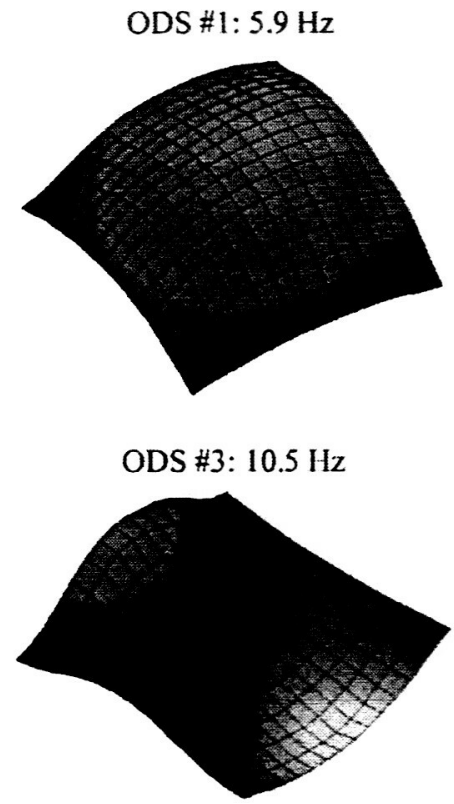

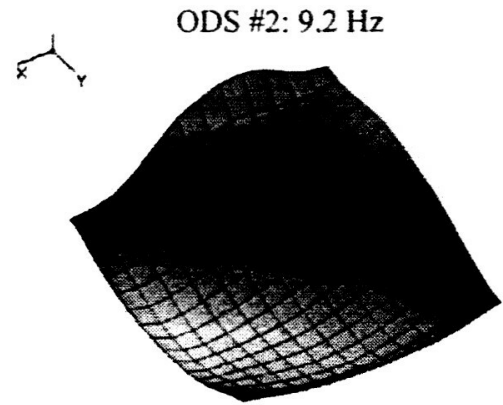

ODS $\# 4: 15.0 \mathrm{~Hz}$

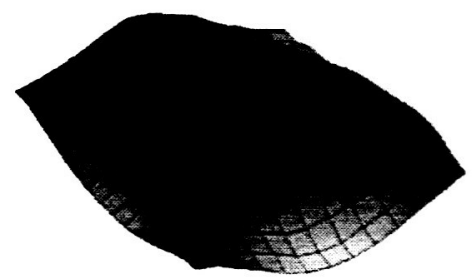

Fig. 16. The first four ODSs and natural frequencies of the rectangular membrane with $T=1.5 \mathrm{lbs}$ and an excitation at the lower left corner.
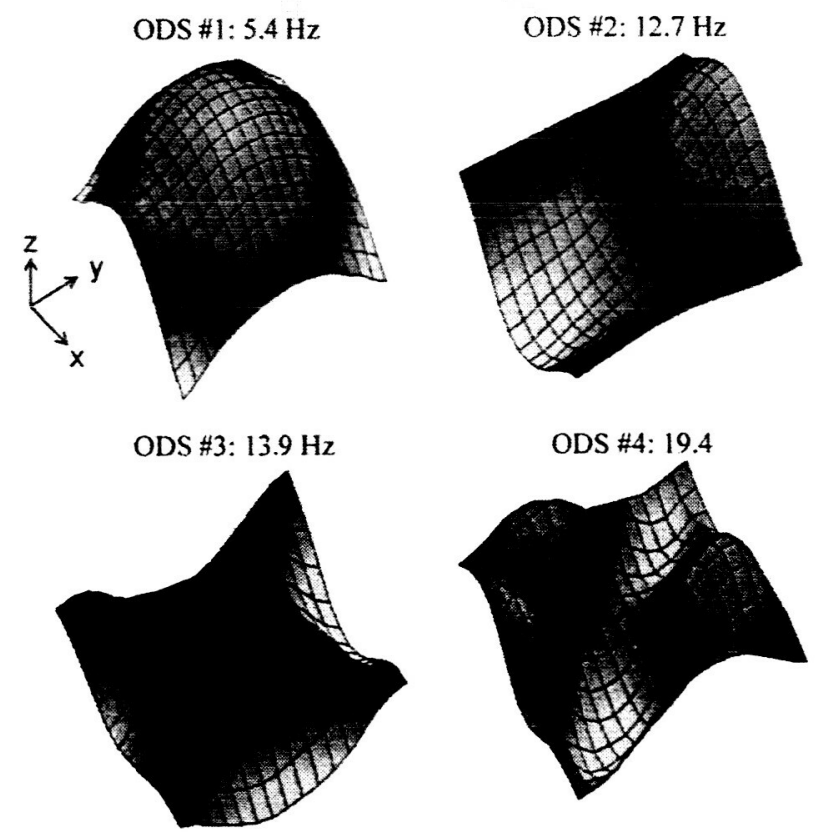

Fig. 17. The first four ODSs and frequencies of the rectangular membrane with $T=1.5 \mathrm{lbs}$ and a simultaneous excitation at four corners. 
483

484

ODSs to be compared with the corresponding numerical mode shapes. The results show that only low-order natural frequencies and ODSs were able to be obtained experimentally because of high structural flexibility, high modal density, severe modal coupling, and heavy air mass. Moreover, experimental natural frequencies are all lower than numerical ones. This discrepancy is attributed to the influence of heavy air mass.

A simple way to account for the air mass effect in the vibration of the rectangular membrane is to increase the membrane's mass density. If the density of the Kapton membrane is increase by a factor of 2.6 to account for air mass when $T=1.5 \mathrm{lbf}$, the numerical natural frequencies of modes \#1, \#6, and \#9 decrease from $13.4,20.0$, and $22.6 \mathrm{~Hz}$ (see Fig. 9) to $8.3,12.4$, and $14.0 \mathrm{~Hz}$, respectively. We note that, although the 6th and 9th numerical natural frequencies become close to the experimental ones in Fig. 17 (ODSs \#2 and \#3), the first natural frequency does not match because the air mass effect is not exactly the same as adding mass to the structure. Hence, advanced analysis by considering the air-membrane interaction needs to be performed in order to accurately predict natural frequencies of membranes in air (Kukathasan and Pellegrino, 2003).

Because the membrane thickness is usually so thin, any significant amplitude of harmonic excitation may cause the vibration amplitude to be larger than the thickness, and non-linear modal coupling, modulation of several linear modes at an unknown frequency, and/or even chaotic vibration may exist (Nayfeh and Pai, 2004). In vibration testing using a scanning laser vibrometer, if the structural vibration is steady and periodic with a known period $T$ and the recording at each location is controlled by triggering to begin at $n T$ ( $n$ is an integer) after the beginning recording time of the previous measurement point, the actual velocity profile at time $t=t_{k}$ will be the distribution of the measured velocities of all points at $n T+t_{k}$, where $n$ is different for each point. The ODS corresponding to the velocity profile can be calculated as the velocity profile divided by $\Omega(=2 \pi / T)$ only if the vibration is harmonic. Hence a scanning laser vibrometer cannot measure a transient ODS or even a steady-state ODS having an unknown period. Fortunately a camera-based motion analysis system can solve these problems because it simultaneously traces all markers and provides truly Lagrangian descriptions of particle motions.

To check whether the ODSs shown in Figs. 16 and 17 are real ODSs we also used our new EAGLE-500 real-time motion analysis system shown in Fig. 18 to measure the ODSs of the membrane with $T=1.5 \mathrm{lbf}$. In order to have non-localized and non-contact excitations we used a lightweight $18 \mathrm{in} . \times 18.25 \mathrm{in} . \times 0.09 \mathrm{in}$. composite plate set-up to be parallel to the membrane and at $2.5 \mathrm{in}$. away from the membrane's center to push the surrounding air to excite the membrane. The composite plate was fixed on a Ling Dynamic LDS V408 shaker, and the excitation amplitude was controlled to be $3.5 \mathrm{~mm}, 4.5 \mathrm{~mm}$, and $5.0 \mathrm{~mm}$ for ODSs \#1, $\# 2$, and \#3, respectively. Experiments showed that this is a very efficient way of exciting the membrane because the air mass effect is so significant. The motion analysis system uses 8 high-resolution CMOS (complementary metal-oxide-semiconductor) cameras to capture pictures of a structure when 8 visible red LED strobes light up retro-reflective markers on the structure. The cameras and strobes are synchronized to work at a speed between 0.1 and 2000 FPS (frames per second). For a frame rate between 0.1 and 480 FPS, a full resolution of $1280 \times 1024$ pixels is used. For a frame rate between 480 and 2000 FPS, a reduced resolution is used. Using triangulation techniques and the known focal lengths (after calibrations using an L-frame with 4 markers and a T-wand with 3 markers) of the cameras and the known coordinates of the bright points (caused by the retro-reflective markers) on the 2D pictures inside the cameras, the EAGLE real-time software EVaRT 4.2 automatically computes and records the instant 3D coordinates of the center of each retro-reflective marker that is seen by at least two cameras. Hence, 3D time traces of all makers are available for performing dynamic animation using stick figures and showing pop-up graphs of displacements, velocities, and accelerations, and they can be output to other programs for further signal processing. The recording time length is effectively infinite and up to 600 markers can be simultaneously traced due to the use of large computer memory and a $100 \mathrm{Mbit}$ data upload rate. Because the $3 \mathrm{D}$ coordinates of each marker are checked and calibrated when more than two cameras see the marker, the measurement accuracy 


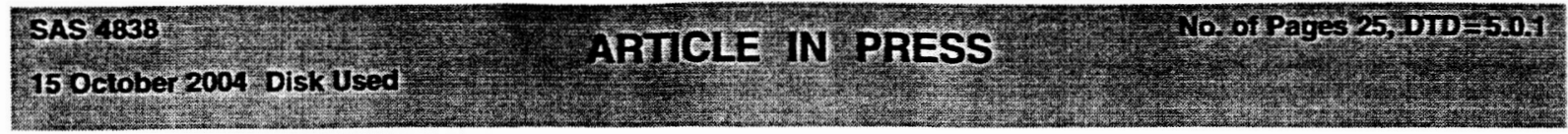

L. G. Young et al. I International Journal of Solids and Structures $x x x(2004) x x x-x x x$

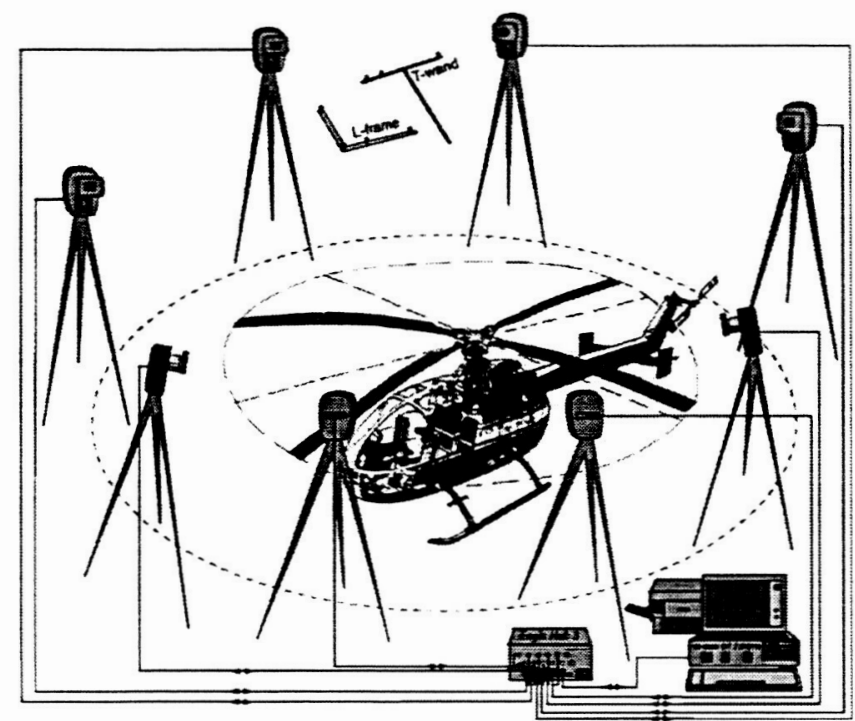

Fig. 18. A typical set-up of an EAGLE-500 motion analysis system for measuring instant coordinates of many markers on a structure.
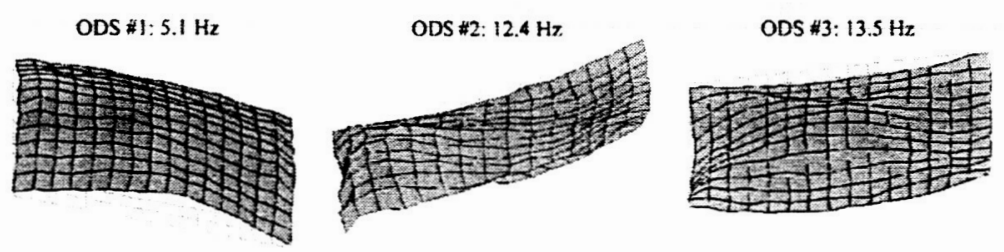

Fig. 19. The first three ODSs and frequencies of the rectangular membrane with $T=1.5 \mathrm{lbs}$ and excited by the surrounding air pushed by a lightweight composite plate.

is high. For example, the measurement error is far less than $1.0 \mathrm{~mm}$ when the measurement volume is $2 \times 2 \times 2 \mathrm{~m}^{3}$. We note that, although the measurement accuracy of the motion analysis system is lower than that of the scanning laser vibrometer, the ODSs measured by the motion analysis system are real ODSs because all points are measured at the same time. Fig. 19 shows the first three ODSs measured by the motion analysis system, which agree well with those in Fig. 17. However, in order to efficiently excite the antisymmetric ODS \#2 shown in Fig. 19, the excitation plate was moved to excite only the left half of the membrane, but the turbulent air flow around the left edge disturbed the ODS shape. Moreover, the ODS \#4 in Fig. 17 could not be obtained, which is probably due to the way of excitation or it is a non-linear mode with internal resonance. This problem requires further studies.

\section{Concluding remarks}

In this paper, we used Jaumann strains and stresses to derive a total-Lagrangian finite-element model of membranes. Results from finite-element analyses of an inflated circular cylindrical Kapton tube and a tensioned rectangular Kapton membrane were verified by experiments using a scanning laser vibrometer and a motion analysis system. Finite-element analyses of the inflated tube showed that shell modes dominate the 
dynamics of the tube, and they are difficult to measure using the scanning laser vibrometer. Finite-element results and experimental data showed that the dynamics of the rectangular membrane is complex. Although some low-frequency mode shapes correlate well with the experimental ones, numerical natural frequencies are far higher than the corresponding experimental ones because of heavy air mass that was not included in the numerical analysis. Moreover, it is almost impossible to verify high-frequency mode shapes because of high modal density, severe modal coupling, and heavy air mass.

\section{Acknowledgments}

The support by the National Science Foundation through Grants CMS-9912482 and CMS-0319853, and the NASA Langley Research Center through Grant NAG-1-01037 is gratefully acknowledged.

\section{References}

ABAQUS, 2001. Standard User's Manual, Version 6.2, Hibbitt, Karlsson, and Sorensen, Inc., Pawtucket, RI.

Bales, G.L., Hall, J.L., Flint, E.M., Glaese, R.M., 2003. Experimental Issues that Impact In-Vacuum Dynamic Characterization of Thin Film Membranes. AIAA-2003-1743.

Choura, S., 1997. Suppression of structural vibrations of an air-inflated membrane dam by its internal pressure. Comput. Struct. 65 (5), 669-677.

Damle, S.V., Joshi, M.N., Sreenivasan, S., Subba Rao, J.V., 1997. Development of cost effective balloon design and fabrication methods. In: AIAA International Balloon Technology Conference, San Francisco, CA, 3-5 June.

Dornhiem, M.A., Anselmo, J.C., 1996. Complex antenna is star of mission 77. Aviation Week Space Technol. 58-59 (May 27).

Gaspar, J.L., Solter, M.J., Pappa, R.S., 2002. Membrane vibration studies using a scanning laser vibrometer. NASA Technical Memorandum, 2002-211427.

Giraudeau, A., Pierron, F., Chambard, J.P., 2002. Experimental study of air effect on vibrating lightweight structures. In: SEM Annual Conference, Milwaukee, Wisconsin, 10-12 June.

Greschik, G., White, C.V., Salama, M.A., 2003. On the precisely uniform and uniaxial tensioning of a film sheet via integrated catenary. AIAA-2003-1906.

Hall, J., Glaese, R.M., Flint, E.M., 2002. Dynamic behavior of thin film membrane strips. In: 43rd AIAA/ASME/ASCE/AHS/ASC Structures, Structural Dynamics, and Materiais Conference, Denver, CO, Aprii.

Holland, D.B., Virgin, L.N., Belvin, W.K., 2003. Investigation of structural dynamics in a 2-meter square solar sail model including axial load effects. AIAA-2003-1746.

Hsieh, J.C., Plaut, R.H., 1990. Free vibration of inflatable dams. Acta Mech. 85, 207-220.

Jacobson, M.B., Iwasa, T., Natori, M.C., 2004. Quantifying square membrane wrinkle behavior using MITC shell elements. AIAA2004-1741.

Jenkins, C.H.M., 2001. Gossamer Spacecraft: Membrane and Inflatable Structures Technology for Space Applications. AIAA, Inc., Reston, VI.

Jha, A.K., Inman, D.J., 2003. Modeling pressure for dynamic analysis of an inflatable structures. AIAA-2003-1833.

Johnson, J., Lienard, S., 2001. Modeling and analysis of structural dynamics for a one-tenth scale model NGST sunshield. In: 42nd AIAA Structures, Structural Dynamics, and Materials Conference, Seattle, WA, April.

Johnston, J., Parrish, K., 2003. Thermal-structural analysis of sunshield membranes. AIAA-2003-1904.

Kukathasan, S., Pellegrino, S., 2002. Vibration of prestressed membrane structures in air. In: 43rd AIAA/ASME/ASCE/AHS/ASC Structures, Structural Dynamics, and Materials Conference, Denver, CO, April.

Kukathasan, S., Pellegrino, S., 2003. Nonlinear vibration of wrinkled membranes. AIAA-2003-1747.

Lee, S.H., 1992. MSC/NASTRAN Handbook for Nonlinear Analysis, Version 67. The MacNeal Schwendler Corporation.

Lennon, B.A., Pellegrino, S., 2000. Stability of lobed inflatable structures. AIAA-2000-1728.

Mysore, G.V., Liapis, S.I., 1998. Dynamic analysis of single-anchor inflatable dams. J. Sound Vibrat. 215 (2), $251-272$.

Nayfeh, A.H., Pai, P.F., 2004. Linear and Nonlinear Structural Mechanics. Wiley-Interscience, New York.

Pai, P.F., Palazotto, A.N., 1995. Polar decomposition theory in nonlinear analyses of solids and structures. J. Eng. Mech. 121 (4), 568 581.

Pai, P.F., Young, L.G., 2003. Fully nonlinear modeling and analysis of precision membranes. Int. J. Comput. Eng. Sci. 4 (1), 1-47. Palisoc, A.L., 1994. Inflatable Reflector Development Program, Task 3 Report. L'Garde Technical Report, LTR-94-AP-008. 